\title{
Skeleton Ideals of Certain Graphs, Standard Monomials and Spherical Parking Functions
}

\author{
Chanchal Kumar \\ IISER Mohali, Knowledge City, \\ Sector 81, SAS Nagar, \\ Punjab -140 306, India \\ \{chanchal, mp15003\}@iisermohali.ac.in
}

\author{
Sonica \\ MCM DAV College for Women \\ Sector- 36 A, \\ Chandigarh - 160 036, India \\ sonica. anand@gmail.com
}

Submitted: Sep 20, 2020; Accepted: Jan 29, 2021; Published: Mar 26, 2021

(C) The authors. Released under the CC BY-ND license (International 4.0).

\begin{abstract}
Let $G$ be a graph on the vertex set $V=\{0,1, \ldots, n\}$ with root 0 . Postnikov and Shapiro were the first to consider a monomial ideal $\mathcal{M}_{G}$, called the $G$-parking function ideal, in the polynomial ring $R=\mathbb{K}\left[x_{1}, \ldots, x_{n}\right]$ over a field $\mathbb{K}$ and explained its connection to the chip-firing game on graphs. The standard monomials of the Artinian quotient $\frac{R}{\mathcal{M}_{G}}$ correspond bijectively to $G$-parking functions. Dochtermann introduced and studied skeleton ideals of the graph $G$, which are subideals of the $G$ parking function ideal with an additional parameter $k(0 \leqslant k \leqslant n-1)$. A $k$-skeleton ideal $\mathcal{M}_{G}^{(k)}$ of the graph $G$ is generated by monomials corresponding to non-empty subsets of the set of non-root vertices $[n]$ of size at most $k+1$. Dochtermann obtained many interesting homological and combinatorial properties of these skeleton ideals. In this paper, we study the $k$-skeleton ideals of graphs and for certain classes of graphs provide explicit formulas and combinatorial interpretation of standard monomials and the Betti numbers.
\end{abstract}

Mathematics Subject Classifications: 05E40, 13D02

\section{Introduction}

Let $G$ be a graph on the vertex set $V=\{0,1, \ldots, n\}$ with a root 0 . The graph $G$ is completely determined by a symmetric $(n+1) \times(n+1)$ matrix $A(G)=\left[a_{i j}\right]_{0 \leqslant i, j \leqslant n}$, called its adjacency matrix, where $a_{i j}$ is the number of edges from $i$ to $j$. Let $R=\mathbb{K}\left[x_{1}, \ldots, x_{n}\right]$ be the standard polynomial ring in $n$ variables over a field $\mathbb{K}$. The $G$-parking function ideal $\mathcal{M}_{G}$ of $G$ is a monomial ideal in $R$ given by the generating set

$$
\mathcal{M}_{G}=\left\langle m_{A}: \emptyset \neq A \subseteq[n]=\{1, \ldots, n\}\right\rangle,
$$


where $m_{A}=\prod_{i \in A} x_{i}^{d_{A}(i)}$ and $d_{A}(i)=\sum_{j \in V \backslash A} a_{i j}$ is the number of edges from $i$ to a vertex outside the set $A$ in $G$. The standard monomial basis $\left\{\mathbf{x}^{\mathbf{b}}=\prod_{i=1}^{n} x_{i}^{b_{i}}\right\}$ of the Artinian quotient $\frac{R}{\mathcal{M}_{G}}$ is determined by the set

$$
\operatorname{PF}(G)=\left\{\mathbf{b}=\left(b_{1}, \ldots, b_{n}\right) \in \mathbb{N}^{n}: \mathbf{x}^{\mathbf{b}} \notin \mathcal{M}_{G}\right\}
$$

of $G$-parking functions. Further, $\operatorname{dim}_{\mathbb{K}}\left(\frac{R}{\mathcal{M}_{G}}\right)$ is the number of spanning trees of $G$, given by the determinant $\operatorname{det}\left(L_{G}\right)$ of the reduced Laplacian matrix $L_{G}$ of $G$. Let $\operatorname{SPT}(G)$ be the set of spanning trees of $G$. The edges of a spanning tree of $G$ are given orientation so that all paths in the spanning tree are directed away from the root. As $|\mathrm{PF}(G)|=|\operatorname{SPT}(G)|$, one would like to construct an explicit bijection $\phi: \operatorname{PF}(G) \rightarrow \operatorname{SPT}(G)$. Using the DepthFirst-Search version of burning algorithm, an algorithmic bijection $\phi: \operatorname{PF}(G) \rightarrow \operatorname{SPT}(G)$ for simple graphs $G$, preserving reverse sum $\operatorname{rsum}(\mathcal{P})$ of $G$-parking function $\mathcal{P}$ and the number $\kappa(G, \phi(\mathcal{P}))$ of $\kappa$-inversions of the spanning tree $\phi(\mathcal{P})$, is constructed by Perkinson, Yang and $\mathrm{Yu}$ [13]. A similar bijection for multigraphs $G$ is constructed by Gaydarov and Hopkins [5].

Postnikov and Shapiro [15] introduced the $G$-parking function ideal $\mathcal{M}_{G}$ and derived many of its combinatorial and homological properties. In particular, they showed that the cellular free complex supported on the first barycentric subdivision $\operatorname{Bd}\left(\Delta_{n-1}\right)$ of an $(n-1)$-simplex $\Delta_{n-1}$ is a free resolution of $\mathcal{M}_{G}$. Further, the cellular resolution of $\mathcal{M}_{G}$ is minimal, provided the graph $G$ is saturated (i.e., $a_{i j}>0$ for $i \neq j$ ). The minimal resolution of the parking function ideal $\mathcal{M}_{G}$ for any graph $G$ is described in $[2,10,12]$.

In a series of papers, Dochtermann $[3,4]$ introduced and studied subideals of the $G$-parking function ideal $\mathcal{M}_{G}$ described by $k$-dimensional 'skeleta'. For an integer $k$ $(0 \leqslant k \leqslant n-1)$, the $k$-skeleton ideal $\mathcal{M}_{G}^{(k)}$ of the graph $G$ is defined as the subideal

$$
\mathcal{M}_{G}^{(k)}=\left\langle m_{A}: \emptyset \neq A \subseteq[n] ;|A| \leqslant k+1\right\rangle
$$

of the monomial ideal $\mathcal{M}_{G}$. For $k=0$, the ideal $\mathcal{M}_{G}^{(0)}$ is generated by powers of variables $x_{1}, \ldots, x_{n}$. Hence, its minimal free resolution and the number of standard monomials can be easily determined. For $k=1$ and $G=K_{n+1}$, the minimal resolution of the one-skeleton ideal $\mathcal{M}_{K_{n+1}}^{(1)}$ is a cocellular resolution supported on the labelled polyhedral complex induced by any generic arrangement of two tropical hyperplanes in $\mathbb{R}^{n}$ and the $i^{\text {th }}$ Betti number

$$
\beta_{i}\left(\frac{R}{\mathcal{M}_{K_{n+1}}^{(1)}}\right)=\sum_{j=1}^{n} j\left(\begin{array}{l}
j-1 \\
i-1
\end{array}\right) \quad \text { for } \quad 1 \leqslant i \leqslant n-1
$$

(see [3]). Also, the number of standard monomials of $\frac{R}{\mathcal{M}_{K_{n+1}}^{(1)}}$ is given by

$$
\operatorname{dim}_{\mathbb{K}}\left(\frac{R}{\mathcal{M}_{K_{n+1}}^{(1)}}\right)=(2 n-1)(n-1)^{n-1}=\operatorname{det}\left(Q_{K_{n+1}}\right),
$$


where $Q_{K_{n+1}}$ is the reduced signless Laplacian matrix of $K_{n+1}$.

In this paper, we determine all the Betti numbers of the $k$-skeleton ideal $\mathcal{M}_{K_{n+1}}^{(k)}$ of the complete graph $K_{n+1}$. The crucial observation is an identification of the ideal $\mathcal{M}_{K_{n+1}}^{(k)}$ with an Alexander dual of some multipermutohedron ideal. We first describe a permutohedron and an associated permutohedron ideal. Let $\mathbf{u}=\left(u_{1}, u_{2}, \ldots, u_{n}\right) \in \mathbb{N}^{n}$ such that $u_{1}<u_{2}<\cdots<u_{n}$ and let $\mathfrak{S}_{n}$ be the set of permutations of $[n]$. For a permutation $\sigma$ of $[n]$, let $\sigma \mathbf{u}=\left(u_{\sigma(1)}, \ldots, u_{\sigma(n)}\right)$ and $\mathbf{x}^{\sigma \mathbf{u}}=\prod_{i=1}^{n} x_{i}^{u_{\sigma(i)}}$. The convex hull of all permutations $\sigma \mathbf{u}$ of $\mathbf{u}$ in $\mathbb{R}^{n}$ is an $(n-1)$-dimensional polytope $\mathbf{P}(\mathbf{u})$, called a permutohedron. Also, the monomial ideal $I(\mathbf{u})=\left\langle\mathbf{x}^{\sigma \mathbf{u}}: \sigma \in \mathfrak{S}_{n}\right\rangle$ of $R$ is called a permutohedron ideal. If some coordinates of $\mathbf{u}=\left(u_{1}, u_{2}, \ldots, u_{n}\right)$ are allowed to be equal, then the polytope $\mathbf{P}(\mathbf{u})$ is called a multipermutohedron and the monomial ideal $I(\mathbf{u})$ is called a multipermutohedron ideal.

The multigraded Betti numbers of multipermutohedron ideals are described in [7]. Also, a combinatorial description of multigraded Betti numbers of Alexander duals of multipermutohedron ideals is given in [8]. Now from the identification of $\mathcal{M}_{K_{n+1}}^{(k)}$ with an Alexander dual of some multipermutohedron ideal, we obtain a combinatorial expression for the $(i-1)^{t h}$ Betti number $\beta_{i-1}\left(\mathcal{M}_{K_{n+1}}^{(k)}\right)$ (Theorem 12). In particular, for $n \geqslant 3$, we show that $\beta_{i-1}\left(\mathcal{M}_{K_{n+1}}^{(1)}\right)=i\left(\begin{array}{c}n+1 \\ i+1\end{array}\right)$ and $\beta_{i-1}\left(\mathcal{M}_{K_{n+1}}^{(n-2)}\right)$ as in Corollary 13.

The main object of study in this paper are spherical $G$-parking functions. A finite sequence $\mathcal{P}=\left(p_{1}, \ldots, p_{n}\right) \in \mathbb{N}^{n}$ is called a $G$-parking function if $\mathbf{x}^{\mathcal{P}}=\prod_{i=1}^{n} x_{i}^{p_{i}} \notin \mathcal{M}_{G}$, on the other hand, the sequence $\mathcal{P}=\left(p_{1}, \ldots, p_{n}\right)$ is called a spherical $G$-parking function if $\mathbf{x}^{\mathcal{P}} \in \mathcal{M}_{G} \backslash \mathcal{M}_{G}^{(n-2)}$. A $G$-parking or a spherical $G$-parking function $\mathcal{P}=\left(p_{1}, \ldots, p_{n}\right) \in \mathbb{N}^{n}$ can be equivalently thought of as a function $\mathcal{P}:[n] \rightarrow \mathbb{N}$ with $\mathcal{P}(i)=p_{i} \quad(1 \leqslant i \leqslant n)$. The sum (or degree) of $\mathcal{P}$ is given by $\operatorname{sum}(\mathcal{P})=\sum_{i \in[n]} \mathcal{P}(i)$. Let

$$
\operatorname{PF}(G)=\left\{\mathcal{P} \in \mathbb{N}^{n}: \mathbf{x}^{\mathcal{P}} \notin \mathcal{M}_{G}\right\} \quad \text { and } \quad \operatorname{sPF}(G)=\left\{\mathcal{P} \in \mathbb{N}^{n}: \mathbf{x}^{\mathcal{P}} \in \mathcal{M}_{G} \backslash \mathcal{M}_{G}^{(n-2)}\right\}
$$

be the sets of $G$-parking functions and spherical $G$-parking functions, respectively. The standard monomials of $\frac{R}{\mathcal{M}_{G}^{(n-2)}}$ are of the form $\mathbf{x}^{\mathcal{P}}$ for $\mathcal{P} \in \operatorname{PF}(G)$ or $\mathcal{P} \in \operatorname{sPF}(G)$. Thus,

$$
\operatorname{dim}_{\mathbb{K}}\left(\frac{R}{\mathcal{M}_{G}^{(n-2)}}\right)=\operatorname{dim}_{\mathbb{K}}\left(\frac{R}{\mathcal{M}_{G}}\right)+\operatorname{dim}_{\mathbb{K}}\left(\frac{\mathcal{M}_{G}}{\mathcal{M}_{G}^{(n-2)}}\right)=|\operatorname{PF}(G)|+|\operatorname{sPF}(G)| .
$$

A notion of spherical $K_{n+1}$-parking functions is introduced in [4]. We recall that a $K_{n+1}$-parking function $\mathcal{P}=\left(p_{1}, \ldots, p_{n}\right) \in \mathbb{N}^{n}$ is an ordinary parking function of length $n$, i.e., a non-decreasing rearrangement $p_{i_{1}} \leqslant p_{i_{2}} \leqslant \cdots \leqslant p_{i_{n}}$ of $\mathcal{P}=\left(p_{1}, \ldots, p_{n}\right)$ satisfies $p_{i_{j}}<j$, for all $j$. It can be easily checked that $\mathcal{P}=\left(p_{1}, \ldots, p_{n}\right) \in \mathbb{N}^{n}$ is a spherical $K_{n+1}$-parking function if a non-decreasing rearrangement $p_{i_{1}} \leqslant p_{i_{2}} \leqslant \cdots \leqslant p_{i_{n}}$ of $\mathcal{P}=$ $\left(p_{1}, \ldots, p_{n}\right)$ satisfies $p_{i_{1}}=1$ and $p_{i_{j}}<j$ for $2 \leqslant j \leqslant n$. The notion of spherical $K_{n+1^{-}}$ parking function has appeared earlier in the literature (see [16]) as prime parking functions of length $n$. Prime parking functions were defined and enumerated by Ira Gessel. The number of spherical $K_{n+1}$-parking functions is $(n-1)^{n-1}$, which is same as the number of 
uprooted trees on the vertex set $[n]$. A (labelled) rooted tree $T$ on the vertex set $[n]$ is called uprooted if the root is bigger than all its children. Let $\mathcal{U}_{n}$ be the set of uprooted trees on the vertex set $[n]$. Dochtermann conjectured existence of a bijection $\phi_{n}: \operatorname{sPF}\left(K_{n+1}\right) \rightarrow \mathcal{U}_{n}$ such that $\operatorname{sum}(\mathcal{P})=\left(\begin{array}{l}n \\ 2\end{array}\right)-\kappa\left(K_{n}, \phi_{n}(\mathcal{P})\right)+1$, where $\kappa\left(K_{n}, \phi_{n}(\mathcal{P})\right)$ is the $\kappa$-number of the uprooted tree $\phi_{n}(\mathcal{P})$ in the complete graph $K_{n}=K_{n+1}-\{0\}$ on the vertex set $[n]$.

For a simple graph $G$ on the vertex set $V$ whose root 0 is connected to all other vertices, we construct an injective map $\phi_{G}: \operatorname{sPF}(G) \rightarrow \mathcal{U}\left(G^{\prime}\right)$, where $G^{\prime}=G-\{0\}$ and $\mathcal{U}\left(G^{\prime}\right)$ is the set of uprooted spanning trees of $G^{\prime}$. Moreover, the injective map $\phi_{G}$ satisfies

$$
\operatorname{sum}(\mathcal{P})=g(G)-\kappa\left(G^{\prime}, \phi_{G}(\mathcal{P})\right)+1 \quad \text { for all } \quad \mathcal{P} \in \operatorname{sPF}(G)
$$

where $g(G)$ is the genus of the graph $G$ (Theorem 20). We have determined the image of $\phi_{G}$ for many simple graphs $G$. In particular, we show that the map $\phi_{K_{n+1}}=\phi_{n}$ : $\operatorname{sPF}\left(K_{n+1}\right) \rightarrow \mathcal{U}_{n}$ is a bijection and establish a conjecture of Dochtermann on spherical $K_{n+1}$-parking functions.

If $e$ is an edge of $G$, then $G-\{e\}$ is the graph obtained from $G$ by deleting the edge $e$. We show that $|\operatorname{sPF}(G)|=\left|\operatorname{sPF}\left(G-\left\{e_{0}\right\}\right)\right|$ (Lemma 17), where $e_{0}$ is an edge from the root to another vertex. As an application, we observe that $\left|\operatorname{sPF}\left(K_{m+1, n}\right)\right|=\left|\operatorname{sPF}\left(K_{n+1, m}\right)\right|$ for complete bipartite graphs (Proposition 33). If $e_{1}$ is an edge in the complete graph $K_{n+1}$, not through the root, we show that $\left|\operatorname{sPF}\left(K_{n+1}-\left\{e_{1}\right\}\right)\right|=(n-1)^{n-3}(n-2)^{2}$ (Theorem $31)$. In this case, spherical $\left(K_{n+1}-\left\{e_{1}\right\}\right)$-parking functions correspond bijectively with some specified subset of uprooted trees on the vertex set $[n]$ (Theorem 23).

Some extensions of these results for the complete multigraph $K_{n+1}^{a, b}$ and the complete bipartite multigraph $K_{m+1, n}^{a, b}(a, b \geqslant 1)$ are also obtained.

Remark 1. This paper is motivated by [3] and an earlier version of [4] posted on the arXiv. In the new version of [4], Dochtermann and King identify the standard monomials of $k$ skeleton ideals $\mathcal{M}_{K_{n+1}}^{(k)}$ with the vector parking functions and using a Breadth-First-Search burning algorithm, they construct a bijection from spherical $K_{n+1}$-parking functions to uprooted spanning trees of $K_{n}$ that takes degree to an inversion statistic. In this paper, we obtain the standard monomials and the Betti numbers of $\mathcal{M}_{K_{n+1}}^{(k)}$ by identifying it with an Alexander dual of some multipermutohedron ideal. For constructing bijection, we use a Depth-First-Search variant of burning algorithm.

\section{Parking functions and Depth-First-Search algorithms}

In this section, we briefly describe some known results on parking functions and the DepthFirst-Search algorithms. Most of the known results are stated without proof. These results and notions will be used in the subsequent sections of this paper.

\subsection{Parking functions}

A sequence $\mathcal{P}=\left(p_{1}, \ldots, p_{n}\right) \in \mathbb{N}^{n}$ is called an ordinary parking function of length $n$, if a non-decreasing rearrangement $p_{i_{1}} \leqslant p_{i_{2}} \leqslant \cdots \leqslant p_{i_{n}}$ of $\mathcal{P}$ satisfies $p_{i_{j}}<j$ for $1 \leqslant j \leqslant n$. 
We denote the set of ordinary parking functions of length $n$ by $\operatorname{PF}(n)$. The notion of ordinary parking function has a nice generalization.

Definition 2. Let $\lambda=\left(\lambda_{1}, \ldots, \lambda_{n}\right) \in \mathbb{N}^{n}$ with $\lambda_{1} \geqslant \lambda_{2} \geqslant \cdots \geqslant \lambda_{n} \geqslant 1$. A finite sequence $\mathcal{P}=\left(p_{1}, \ldots, p_{n}\right) \in \mathbb{N}^{n}$ is called a $\lambda$-parking function of length $n$, if a non-decreasing rearrangement $p_{i_{1}} \leqslant p_{i_{2}} \leqslant \cdots \leqslant p_{i_{n}}$ of $\mathcal{P}$ satisfies $p_{i_{j}}<\lambda_{n-j+1}$ for $1 \leqslant j \leqslant n$. Let $\operatorname{PF}(\lambda)$ be the set of $\lambda$-parking functions.

Clearly, the ordinary parking functions of length $n$ are precisely $\lambda$-parking functions of length $n$ for $\lambda=(n, n-1, \ldots, 2,1) \in \mathbb{N}^{n}$. The number of $\lambda$-parking functions is given by the 'so-called' Steck determinantal formula (see [14]). Let

$$
\Lambda\left(\lambda_{1}, \ldots, \lambda_{n}\right)=\left[\frac{\lambda_{n-i+1}^{j-i+1}}{(j-i+1) !}\right]_{1 \leqslant i, j \leqslant n} .
$$

In other words, the $(i, j)^{t h}$ entry of the $n \times n$ matrix $\Lambda\left(\lambda_{1}, \ldots, \lambda_{n}\right)$ is $\frac{\lambda_{n-i+1}^{j-i+1}}{(j-i+1) !}$, where by convention, $\frac{1}{(j-i+1) !}=0$ for $i>j+1$. The determinant $\operatorname{det}\left(\Lambda\left(\lambda_{1}, \ldots, \lambda_{n}\right)\right)$ is called a Steck determinant.

Theorem 3 (Pitman-Stanley). The number of $\lambda$-parking functions is given by

$$
|\operatorname{PF}(\lambda)|=(n !) \operatorname{det}\left(\Lambda\left(\lambda_{1}, \ldots, \lambda_{n}\right)\right)=n ! \quad \operatorname{det}\left[\frac{\lambda_{n-i+1}^{j-i+1}}{(j-i+1) !}\right]_{1 \leqslant i, j \leqslant n} .
$$

For $\lambda=\left(\lambda_{1}, \ldots, \lambda_{n}\right) \in \mathbb{N}^{n}$ with $\lambda_{1} \geqslant \lambda_{2} \geqslant \cdots \geqslant \lambda_{n} \geqslant 1$, Postnikov and Shapiro [15] considered the monomial ideal

$$
\mathcal{M}_{\lambda}=\left\langle\left(\prod_{j \in A} x_{j}\right)^{\lambda_{|A|}}: \emptyset \neq A \subseteq[n]\right\rangle
$$

in the polynomial ring $R=\mathbb{K}\left[x_{1}, \ldots, x_{n}\right]$. A monomial $\mathbf{x}^{\mathbf{b}}=\prod_{j=1}^{n} x_{j}^{b_{j}} \notin \mathcal{M}_{\lambda}$ is called a standard monomial of $\frac{R}{\mathcal{M}_{\lambda}}$ or $\mathcal{M}_{\lambda}$. Clearly, $\mathbf{x}^{\mathbf{b}}=\prod_{j=1}^{n} x_{j}^{b_{j}}$ is a standard monomial of $\mathcal{M}_{\lambda}$ if and only if $\mathbf{b}=\left(b_{1}, \ldots, b_{n}\right) \in \operatorname{PF}(\lambda)$. In other words, a monomial basis of the $\mathbb{K}$-vector space $\frac{R}{\mathcal{M}_{\lambda}}$ correspond bijectively with the $\lambda$-parking functions.

Theorem 4 (Pitman-Stanley, Postnikov-Shapiro). The dimension of $\frac{R}{\mathcal{M}_{\lambda}}$ is given by

$$
\operatorname{dim}_{\mathbb{K}}\left(\frac{R}{\mathcal{M}_{\lambda}}\right)=|\operatorname{PF}(\lambda)|=\sum_{\left(b_{1}, \ldots, b_{n}\right) \in \operatorname{PF}(n)} \prod_{i=1}^{n}\left(\lambda_{n-b_{i}}-\lambda_{n-b_{i}+1}\right),
$$

where the summation runs over ordinary parking functions of length $n$ and $\lambda_{n+1}=0$.

A closed formula for the number of $\lambda$-parking functions for various specific values of $\lambda$ is given in $[14,17]$. For more on parking functions, we refer to an excellent survey article by Yan [18]. 


\subsection{Graph theoretic notions and $G$-parking functions}

Let $G$ be a connected graph on the vertex set $V(G)=V=\{0,1, \ldots, n\}$. Suppose $A(G)=\left[a_{i j}\right]_{0 \leqslant i, j \leqslant n}$ is the (symmetric) adjacency matrix of $G$. We assume that $G$ is a loopless graph, i.e., $a_{i i}=0$ for all $i$. Let $E(i, j)=E(j, i)$ be the set of edges between distinct pair of vertices $i, j \in V$. If $E(i, j) \neq \emptyset$, then $i$ and $j$ are called adjacent vertices and we write $i \sim j$. On the other hand, if $i$ and $j$ are non-adjacent, we write $i \nsim j$. We have $|E(i, j)|=a_{i j}$. The graph $G$ is called a simple graph if $|E(i, j)|=a_{i j} \leqslant 1$ for $i, j \in V$. Otherwise, $G$ is called a multigraph. The set $E(G)=\bigcup_{i, j \in V} E(i, j)$ is the set of edges of $G$.

If $v \in V$, then $G-\{v\}$ denotes the graph on the vertex set $V \backslash\{v\}$ obtained from $G$ by deleting the vertex $v$ and all the edges through $v$. If $e \in E(G)$ is an edge of $G$, then $G-\{e\}$ denotes the graph on the vertex set $V$ obtained from $G$ by deleting the edge $e$. If $E(i, j) \neq \emptyset$, then $G-E(i, j)$ denotes the graph on vertex set $V$ obtained from $G$ on deleting all the edges between $i$ and $j$.

Fix a root $r \in V$ of $G$ (usually, we take $r=0$ ). Set $\widetilde{V}=V \backslash\{r\}$. Let $\operatorname{SPT}(G)$ be the set of spanning trees of $G$ rooted at $r$. We orient spanning tree $T \in \operatorname{SPT}(G)$ so that all paths in $T$ are directed away from the root $r$. For every $j \in \widetilde{V}$, there is a unique oriented path in $T$ from the root $r$ to $j$. An $i \in \widetilde{V}$ lying on this unique path in $T$ is called an ancestor of $j$ in $T$. Equivalently, we say that $j$ is a descendent of $i$ in $T$. If in addition, $i$ and $j$ are adjacent in $T$, then we say that $i$ is a parent of its child $j$. Every child $j$ has a unique parent $\operatorname{par}_{T}(j)$ in $T$.

Definition 5. By an inversion of $T \in \operatorname{SPT}(G)$, we mean an ordered pair $(i, j)$ of vertices such that $i$ is an ancestor of $j$ in $T$ with $i>j$. The total number of inversions of a spanning tree $T$ is denoted by $\operatorname{inv}(T)$. An inversion $(i, j)$ of $T$ is called a $\kappa$-inversion of $T$ if $i$ is not the root $r$ and $\operatorname{par}_{T}(i)$ is adjacent to $j$ in $G$.

The invariant $g(G)=|E(G)|-|V(G)|+1$ is called the genus of the graph $G$. The $\kappa$-number $\kappa(G, T)$ of $T$ in $G$ is given by

$$
\kappa(G, T)=\sum_{\substack{i, j \in \widetilde{V} \\ i>j}}\left|E\left(\operatorname{par}_{T}(i), j\right)\right| .
$$

For a simple graph $G$, the total number of $\kappa$-inversions of $T$ is $\kappa(G, T)$. If $G=K_{n+1}$ with root 0 , then $\kappa\left(K_{n+1}, T\right)=\operatorname{inv}(T)$ for every $T \in \operatorname{SPT}\left(K_{n+1}\right)$.

Definition 6. Let $G$ be a graph on the vertex set $V=\{0,1, \ldots, n\}$ with the adjacency matrix $A(G)=\left[a_{i j}\right]_{0 \leqslant i, j \leqslant n}$. Let $r \in V$ be the root of $G$ and $\widetilde{V}=V \backslash\{r\}$. A function $\mathcal{P}: \widetilde{V} \rightarrow \mathbb{N}$ is called a $G$-parking function (with respect to the root $r$ ) if for every nonempty set $A \subseteq \widetilde{V}$, there exists $i \in A$ such that $\mathcal{P}(i)<d_{A}(i)=\sum_{j \in V \backslash A} a_{i j}$.

Note that, if root $r=0$, then $\mathcal{P}$ is a $G$-parking function if and only if $\mathbf{x}^{\mathcal{P}} \notin \mathcal{M}_{G}$, i.e., $\mathbf{x}^{\mathcal{P}}$ is a standard monomial of the $G$-parking function ideal $\mathcal{M}_{G}$. For a $G$-parking 
function $\mathcal{P}: \widetilde{V} \rightarrow \mathbb{N}$, the $\operatorname{sum} \operatorname{sum}(\mathcal{P})$ and the reverse $\operatorname{sum} \operatorname{rsum}(\mathcal{P})$ of $\mathcal{P}$ are respectively given by

$$
\operatorname{sum}(\mathcal{P})=\sum_{i \in \widetilde{V}} \mathcal{P}(i) \quad \text { and } \quad \operatorname{rsum}(\mathcal{P})=g(G)-\operatorname{sum}(\mathcal{P})=g(G)-\sum_{i \in \widetilde{V}} \mathcal{P}(i)
$$

Definition 7. A rooted tree on the vertex set $[n]$ is called an uprooted tree if the root is bigger than all its children.

Let $\mathcal{U}_{n}$ be the set of uprooted trees on the vertex set $[n]$. Then it is well known that $\left|\mathcal{U}_{n}\right|=(n-1)^{n-1}$. For certain graphs $G$ on the vertex set $V$, we shall show that the spherical $G$-parking functions correspond to uprooted spanning trees of $G^{\prime}=G-\{0\}$.

\subsection{Depth-First-Search Algorithms}

We now describe the Depth-First-Search burning algorithm of Perkinson-Yang-Yu [13] for simple graphs. Let $G$ be a simple graph on the vertex set $V$ with a root $r \in V$. Applied to an input function $\mathcal{P}: V \backslash\{r\} \rightarrow \mathbb{N}$, the Depth-First-Search algorithm of PerkinsonYang-Yu [13] gives a subset burnt_vertices of burnt vertices and a subset tree_edges of tree edges as an output. We imagine that a fire starts at the root $r$ and spread to other vertices of $G$ according to the depth-first rule. The value $\mathcal{P}(j)$ of the input function $\mathcal{P}$ can be considered as the number of water droplets available at vertex $j$ that prevents spread of fire to $j$. If $i$ is a burnt vertex, then consider the largest non-burnt vertex $j$ adjacent to $i$. If $\mathcal{P}(j)=0$, then fire from $i$ will spread to $j$. In this case, add $j$ in burnt_vertices and include the edge $(i, j)$ in tree_edges. Now the fire spreads from the burnt vertex $j$. On the other hand, if $\mathcal{P}(j)>0$, then one water droplet available at $j$ will be used to prevent fire from reaching $j$ through the edge $(i, j)$. In this case, the dampened edge $(i, j)$ is removed from $G$, number of water droplets available at $j$ is reduced to $\mathcal{P}(j)-1$ and the fire continue to spread from the burnt vertex $i$ through non-dampened edges. If all the edges from $i$ to unburnt vertices get dampened, then the search backtracks. At the start, burnt_vertices $=\{r\}$ and tree_edges $=\{\}$.

Perkinson, Yang and $\mathrm{Yu}[13]$ constructed a bijection $\phi: \operatorname{PF}(G) \rightarrow \operatorname{SPT}(G)$ using their Depth-First-Search algorithm.

Theorem 8 (Perkinson-Yang-Yu). Let $G$ be a simple graph on the vertex set $V$ with root $r$. After applying Depth-First-Search burning algorithm to $\mathcal{P}: V \backslash\{r\} \rightarrow \mathbb{N}$, if burnt_vertices $=V$, then $\mathcal{P}$ is a $G$-parking function and tree edges in the set tree_edges form a spanning tree $\phi(\mathcal{P})$ of $G$. If burnt_vertices $\neq V$, then $\mathcal{P}$ is not a $G$-parking function. Further, the mapping $\mathcal{P} \mapsto \phi(\mathcal{P})$ given by the Depth-First-Search algorithm induces a bijection $\phi: \operatorname{PF}(G) \rightarrow \operatorname{SPT}(G)$ such that

$$
\operatorname{rsum}(\mathcal{P})=g(G)-\operatorname{sum}(\mathcal{P})=\kappa(G, \phi(\mathcal{P})) \quad \text { for all } \quad \mathcal{P} \in \operatorname{PF}(G)
$$


Let $\sum_{\mathcal{P} \in \operatorname{PF}(G)} q^{\text {rsum }(\mathcal{P})}$ be the reversed sum enumerator for $G$-parking functions. Theorem 8 establishes the identity

$$
\sum_{\mathcal{P} \in \operatorname{PF}(G)} q^{\mathrm{rsum}(\mathcal{P})}=\sum_{T \in \operatorname{SPT}(G)} q^{\kappa(G, T)}
$$

that extends a similar identity obtained by Kreweras [6] for the complete graph $K_{n+1}$.

We now describe the Depth-First-Search burning algorithm of Gaydarov-Hopkins [5] for multigraphs. Consider a connected multigraph $G$ on the vertex set $V$ with root $r$. Let $E(i, j)=E(j, i)$ be the set of edges between distinct pair of vertices $i$ and $j$. Fix a total order on $E(i, j)$ for all distinct pairs $\{i, j\}$ of vertices and write $E(i, j)=$ $\left\{e_{i j}^{0}, e_{i j}^{1}, \ldots, e_{i j}^{a_{i j}-1}\right\}$, where $|E(i, j)|=a_{i j}$. Thus we assume that edges of the multigraph $G$ are labelled. Applied to an input function $\mathcal{P}: V \backslash\{r\} \rightarrow \mathbb{N}$, the Depth-First-Search algorithm for multigraphs gives a subset burnt_vertices of burnt vertices and a subset tree_edges of tree edges with nonnegative labels on them as an output. As in the case of Depth-First-Search algorithm for simple graphs, we imagine that a fire starts at the root $r$ and spread to other vertices of $G$ according to the depth-first rule. If $i$ is a burnt vertex, then consider the largest non-burnt vertex $j$ adjacent to $i$. If $\mathcal{P}(j)<a_{i j}=|E(i, j)|$, then $\mathcal{P}(j)$ edges with higher labels, namely $e_{i j}^{a_{i j}-1}, \ldots, e_{i j}^{a_{i j}-\mathcal{P}(j)}$ will get dampened, the edge $e_{i j}^{a_{i j}-\mathcal{P}(j)-1}$ with label $a_{i j}-\mathcal{P}(j)-1$ will be added to tree_edges and $j$ in included in burnt_vertices. Now fire will spread from the burnt vertex $j$. On the other hand, if $\mathcal{P}(j) \geqslant a_{i j}$, then all the edges in $E(i, j)$ get dampened and $\mathcal{P}(j)$ reduced to $\mathcal{P}(j)-a_{i j}$. The fire continue to spread from the burnt vertex $i$ through non-dampened edges. If all the edges from $i$ to unburnt vertices get dampened, then the search backtracks. At the start, burnt_vertices $=\{r\}$ and tree_edges $=\{\}$. Gaydarov and Hopkins [5] extended Theorem 8 to multigraphs using the Depth-First-Search burning algorithm for multigraph.

Theorem 9 (Gaydarov-Hopkins). Let $G$ be a multigraph on $V$ with root $r$. After applying Depth-First-Search burning algorithm to $\mathcal{P}: V \backslash\{r\} \rightarrow \mathbb{N}$, if burnt_vertices $=V$, then $\mathcal{P}$ is a $G$-parking function and tree edges with labels in the set tree_edges form a labelled spanning tree $\phi(\mathcal{P})$ of $G$. If burnt_vertices $\neq V$, then $\mathcal{P}$ is not a $G$-parking function. Suppose $\ell(e)$ is the label on an edge e of $\phi(\mathcal{P})$. Then the mapping $\mathcal{P} \mapsto \phi(\mathcal{P})$ given by Depth-First-Search burning algorithm induces a bijection $\phi: \operatorname{PF}(G) \rightarrow \operatorname{SPT}(G)$ such that

$$
\operatorname{rsum}(\mathcal{P})=\kappa(G, T)+\sum_{e \in E(T)} \ell(e) \quad \text { for all } \quad \mathcal{P} \in \operatorname{PF}(G), \quad \text { where } \quad T=\phi(\mathcal{P}) .
$$

The bijective map induced by the Depth-First-Search algorithms is always denoted by $\phi$ in this paper ignoring its dependence on the graph and the root. 


\section{$3 \quad k$-skeleton ideals of complete graphs}

Let $0 \leqslant k \leqslant n-1$. Consider the $k$-skeleton ideal $\mathcal{M}_{K_{n+1}}^{(k)}$ of the complete graph $K_{n+1}$ on the vertex set $V=\{0,1, \ldots, n\}$. As stated in the Introduction, we have

$$
\mathcal{M}_{K_{n+1}}^{(k)}=\left\langle\left(\prod_{j \in A} x_{j}\right)^{n-|A|+1}: \emptyset \neq A \subseteq[n] ;|A| \leqslant k+1\right\rangle .
$$

For $k=0, \mathcal{M}_{K_{n+1}}^{(0)}=\left\langle x_{1}^{n}, \ldots, x_{n}^{n}\right\rangle$ is a monomial ideal in $R$ generated by $n^{\text {th }}$ power of variables. Thus, its minimal free resolution is given by the Koszul complex associated to the regular sequence $x_{1}^{n}, \ldots, x_{n}^{n}$ in $R$. Also, $\operatorname{dim}_{\mathbb{K}}\left(\frac{R}{\mathcal{M}_{K_{n+1}}^{(0)}}\right)=n^{n}$. For $k=n-1$, $\mathcal{M}_{K_{n+1}}^{(n-1)}=\mathcal{M}_{K_{n+1}}$. The minimal free resolution of the $K_{n+1}$-parking function ideal $\mathcal{M}_{K_{n+1}}$ is the cellular resolution supported on the first barycentric subdivision $\mathbf{B d}\left(\Delta_{n-1}\right)$ of an $n$-1-simplex $\Delta_{n-1}$ and

$$
\operatorname{dim}_{\mathbb{K}}\left(\frac{R}{\mathcal{M}_{K_{n+1}}}\right)=\left|\operatorname{PF}\left(K_{n+1}\right)\right|=\left|\operatorname{SPT}\left(K_{n+1}\right)\right|=(n+1)^{n-1} .
$$

For $k=1$, the 1 -skeleton ideal $\mathcal{M}_{K_{n+1}}^{(1)}$ has a minimal cocellular resolution supported on the labelled polyhedral complex induced by any generic arrangement of two tropical hyperplanes in $\mathbb{R}^{n-1}$ (see Theorem 4.6 of [3]) and $\operatorname{dim}_{\mathbb{K}}\left(\frac{R}{\mathcal{M}_{K_{n+1}}^{(1)}}\right)=(2 n-1)(n-1)^{n-1}$.

\subsection{Betti numbers of $\mathcal{M}_{K_{n+1}}^{(k)}$}

We now express the $k$-skeleton ideal $\mathcal{M}_{K_{n+1}}^{(k)}$ of $K_{n+1}$ as an Alexander dual of a multipermutohedron ideal. Let $\mathbf{u}=\left(u_{1}, u_{2}, \ldots, u_{n}\right) \in \mathbb{N}^{n}$ such that $u_{1} \leqslant u_{2} \leqslant \ldots \leqslant u_{n}$. Set $\mathbf{m}=\left(m_{1}, \ldots, m_{s}\right)$ such that the smallest entry in $\mathbf{u}$ is repeated exactly $m_{1}$ times, second smallest entry in $\mathbf{u}$ is repeated exactly $m_{2}$ times, and so on. Then $\sum_{j=1}^{s} m_{j}=n$ and $m_{j} \geqslant 1$ for all $j$. In this case, we write $\mathbf{u}(\mathbf{m})$ for $\mathbf{u}$. The monomial ideal $I(\mathbf{u}(\mathbf{m}))=$ $\left\langle\mathbf{x}^{\sigma \mathbf{u}(\mathbf{m})}: \sigma \in \mathfrak{S}_{n}\right\rangle$ of $R$ is called a multipermutohedron ideal. If $\mathbf{m}=(1, \ldots, 1) \in \mathbb{N}^{n}$, then $I(\mathbf{u}(\mathbf{m}))$ is a permutohedron ideal.

Let $\mathbf{u}(\mathbf{m})=(1,2, \ldots, k, k+1, \ldots, k+1) \in \mathbb{N}^{n}$, where $\mathbf{m}=(1, \ldots, 1, n-k) \in \mathbb{N}^{k+1}$. For $k=0, \mathbf{u}(\mathbf{m})=(1, \ldots, 1) \in \mathbb{N}^{n}$, while for $k=n-1, \mathbf{u}(\mathbf{m})=(1,2, \ldots, n) \in \mathbb{N}^{n}$. Let $I(\mathbf{u}(\mathbf{m}))^{[\mathbf{n}]}$ be the Alexander dual of the multipermutohedron ideal $I(\mathbf{u}(\mathbf{m}))$ with respect to $\mathbf{n}=(n, \ldots, n) \in \mathbb{N}^{n}$.

Theorem 10. For $0 \leqslant k \leqslant n-1, \mathcal{M}_{K_{n+1}}^{(k)}=I(\mathbf{u}(\mathbf{m}))^{[\mathbf{n}]}$.

Proof. Using Proposition 5.23 of [11], it follows from the Lemma 2.3 of [8].

Let $\mathbf{b}=\left(b_{1}, \ldots, b_{n}\right) \in \mathbb{N}^{n}$. The $(i-1)^{t h}$ multigraded Betti number $\beta_{i-1, \mathbf{b}}\left(\mathcal{M}_{K_{n+1}}^{(k)}\right)$ of $\mathcal{M}_{K_{n+1}}^{(k)}$ in degree $\mathbf{b}$ is given by

$$
\beta_{i-1, \mathbf{b}}\left(\mathcal{M}_{K_{n+1}}^{(k)}\right)=\operatorname{dim}_{\mathbb{K}} \tilde{H}^{|\operatorname{Supp}(\mathbf{b})|-i-1}\left(K_{\mathbf{b}}\left(\mathcal{M}_{K_{n+1}}^{(k)}\right) ; \mathbb{K}\right) \quad \text { for } \quad i \geqslant 1,
$$


where $K_{\mathbf{b}}\left(\mathcal{M}_{K_{n+1}}^{(k)}\right)$ is the lower Koszul simplicial complex of $\mathcal{M}_{K_{n+1}}^{(k)}$ in degree $\mathbf{b}$ and $\operatorname{Supp}(\mathbf{b})=\left\{j: b_{j}>0\right\}$ (see Theorem 5.11 of $[11]$ ). Since $\mathcal{M}_{K_{n+1}}^{(k)}=I(\mathbf{u}(\mathbf{m}))^{[\mathbf{n}]}$, a combinatorial description of all multidegrees $\mathbf{b}$ such that $\beta_{i-1, \mathbf{b}}\left(\mathcal{M}_{K_{n+1}}^{(k)}\right) \neq 0$ is given in terms of dual $\mathbf{m}$-isolated subsets (see Definition 3.1 and Theorem 3.2 of [8]). For the particular case of $\mathbf{m}=(1, \ldots, 1, n-k) \in \mathbb{N}^{k+1}$, the notion of dual $\mathbf{m}$-isolated subsets can be easily described. Let $J=\left\{j_{1}, \ldots, j_{t}\right\} \subseteq[n]$ be a non-empty subset with $0=j_{0}<j_{1}<$ $\cdots<j_{t}$.

1. $J$ is a dual $\mathbf{m}$-isolated subset of type- 1 if $J \subseteq[k+1]$ and its dual weight $\operatorname{dwt}(J)=t-1$. Let $\mathcal{I}_{\mathbf{m}}^{*, 1}$ be the set of dual $\mathbf{m}$-isolated subsets of type- 1 and let $\mathcal{I}_{\mathbf{m}}^{*, 1}\langle i\rangle=\left\{J \in \mathcal{I}_{\mathbf{m}}^{*, 1}\right.$ : $\operatorname{dwt}(J)=i\}$.

2. $J=\left\{j_{1}, \ldots, j_{t}\right\}$ is a dual $\mathbf{m}$ - isolated subset of type-2 if $J \backslash\left\{j_{t}\right\} \subseteq[k], k+1<j_{t} \leqslant n$ and its dual weight $\operatorname{dwt}(J)=(t-2)+\left(j_{t}-k\right)$. Let $\mathcal{I}_{\mathbf{m}}^{*, 2}$ be the set of dual-m isolated subsets of type-2 and let $\mathcal{I}_{\mathbf{m}}^{*, 2}\langle i\rangle=\left\{J \in \mathcal{I}_{\mathbf{m}}^{*, 2}: \operatorname{dwt}(J)=i\right\}$.

Let $\mathcal{I}_{\mathbf{m}}^{*}=\mathcal{I}_{\mathbf{m}}^{*, 1} \coprod \mathcal{I}_{\mathbf{m}}^{*, 2}$ be the set of all dual $\mathbf{m}$-isolated subsets and $\mathcal{I}_{\mathbf{m}}^{*}\langle i\rangle=\mathcal{I}_{\mathbf{m}}^{*, 1}\langle i\rangle \coprod \mathcal{I}_{\mathbf{m}}^{*, 2}\langle i\rangle$.

Consider $\lambda=\left(\lambda_{1}, \lambda_{2}, \ldots, \lambda_{n}\right)$ with $\lambda_{i}=n-i+1$ for $1 \leqslant i \leqslant k$ and $\lambda_{i}=n-k$ for $k+1 \leqslant i \leqslant n$. Let $e_{1}, \ldots, e_{n}$ be the standard basis vectors of $\mathbb{R}^{n}$. For $0 \leqslant i<j \leqslant n$, we set $\varepsilon(i, j)=\sum_{l=i+1}^{j} e_{l}$. For any $J=\left\{j_{1}, \ldots, j_{t}\right\} \in \mathcal{I}_{\mathbf{m}}^{*}$, let $\mathbf{b}(J)=\sum_{\alpha=1}^{t} \lambda_{j_{\alpha}} \varepsilon\left(j_{\alpha-1}, j_{\alpha}\right) \in \mathbb{N}^{n}$.

We illustrate the concept of dual $\mathbf{m}$-isolated subsets and its relation with multigraded Betti numbers with an example.

Example 11. Let $n=6$ and $k=2$. Take $\mathbf{u}(\mathbf{m})=(1,2,3,3,3,3)$. Then $\mathbf{m}=(1,1,4)$ and $\lambda=(6,5,4,4,4,4)$. Consider the multipermutohedron ideal $I(\mathbf{u}(\mathbf{m}))$ and the 2skeleton ideal $\mathcal{M}_{K_{6+1}}^{(2)}$. Set $\mathbf{6}=(6,6,6,6,6,6)$. The Alexander dual $I(\mathbf{u}(\mathbf{m}))^{[\mathbf{6}]}=\mathcal{M}_{K_{6+1}}^{(2)}$. A subset $J \subseteq[3]$ is a dual $\mathbf{m}$-isolated subset of type-1. For example, $J=\{2\}$ and $\tilde{J}=\{1,3\}$ are dual $\mathbf{m}$-isolated subsets of type- 1 with dual weights 0 and 1 , respectively. Also, the associated multidegrees are $\mathbf{b}(J)=(5,5,0,0,0,0)$ and $\mathbf{b}(\tilde{J})=(6,4,4,0,0,0)$. The lower Koszul simplicial complex $K_{\mathbf{b}}\left(\mathcal{M}_{K_{6+1}}^{(2)}\right)$ for $\mathbf{b}=\mathbf{b}(J)$ or $\mathbf{b}(\tilde{J})$ is isomorphic to the 0 -dimensional simplicial complex consisting of two points. Thus $\beta_{0, \mathbf{b}(J)}\left(\mathcal{M}_{K_{6+1}}^{(2)}\right)=1$ and $\beta_{1, \mathbf{b}(\tilde{J})}\left(\mathcal{M}_{K_{6+1}}^{(2)}\right)=1$. Further, the subsets $J^{\prime}=\{4\}$ and $J^{\prime \prime}=\{1,5\}$ are examples of dual $\mathbf{m}$-isolated subsets of type- 2 with dual weights 1 and 3 , respectively. We have $\mathbf{b}\left(J^{\prime}\right)=(4,4,4,4,0,0)$ and $\mathbf{b}\left(J^{\prime \prime}\right)=(6,4,4,4,4,0)$. The lower Koszul simplicial complex $K_{\mathbf{b}\left(J^{\prime}\right)}\left(\mathcal{M}_{K_{6+1}}^{(2)}\right)$ is isomorphic to the 0-skeleton of a 3 -simplex, while $K_{\mathbf{b}\left(J^{\prime \prime}\right)}\left(\mathcal{M}_{K_{6+1}}^{(2)}\right)$ is isomorphic to the 1 -skeleton of a 3 -simplex. Therefore $\beta_{1, \mathbf{b}\left(J^{\prime}\right)}\left(\mathcal{M}_{K_{6+1}}^{(2)}\right)=3$ and $\beta_{3, \mathbf{b}\left(J^{\prime \prime}\right)}\left(\mathcal{M}_{K_{6+1}}^{(2)}\right)=3$.

Theorem 12. For $\mathbf{b}=\left(b_{1}, \ldots, b_{n}\right) \in \mathbb{N}^{n}$ and $1 \leqslant i \leqslant n$, let $\beta_{i-1, \mathbf{b}}\left(\mathcal{M}_{K_{n+1}}^{(k)}\right)$ be the $(i-1)^{\text {th }}$ multigraded Betti number of $\mathcal{M}_{K_{n+1}}^{(k)}$ in degree $\mathbf{b}$. Then the following statements hold.

(i) For $J=\left\{j_{1}, \ldots, j_{t}\right\} \in \mathcal{I}_{\mathbf{m}}^{*, 1}\langle i-1\rangle, \beta_{i-1, \mathbf{b}(J)}\left(\mathcal{M}_{K_{n+1}}^{(k)}\right)=1$, where $t=i$. 
(ii) For $J=\left\{j_{1}, \ldots, j_{t}\right\} \in \mathcal{I}_{\mathbf{m}}^{*, 2}\langle i-1\rangle, \beta_{i-1, \mathbf{b}(J)}\left(\mathcal{M}_{K_{n+1}}^{(k)}\right)=\left(\begin{array}{c}j_{t}-j_{t-1}-1 \\ k-j_{t-1}\end{array}\right)$, where $t+j_{t}-k=$ $i+1$.

(iii) If $\mathbf{b}=\pi \mathbf{b}(J)$ is a permutation of $\mathbf{b}(J)$ for some $J \in \mathcal{I}_{\mathbf{m}}^{*}\langle i-1\rangle$ and some $\pi \in \mathfrak{S}_{n}$, then $\beta_{i-1, \mathbf{b}}\left(\mathcal{M}_{K_{n+1}}^{(k)}\right)=\beta_{i-1, \mathbf{b}(J)}\left(\mathcal{M}_{K_{n+1}}^{(k)}\right)$. Otherwise, $\beta_{i-1, \mathbf{b}}\left(\mathcal{M}_{K_{n+1}}^{(k)}\right)=0$.

(iv) The $(i-1)^{t h}$-Betti number $\beta_{i-1}\left(\mathcal{M}_{K_{n+1}}^{(k)}\right)$ of $\mathcal{M}_{K_{n+1}}^{(k)}$ is given by,

$$
\beta_{i-1}\left(\mathcal{M}_{K_{n+1}}^{(k)}\right)=\beta_{i}\left(\frac{R}{\mathcal{M}_{K_{n+1}}^{(k)}}\right)=\sum_{J \in \mathcal{I}_{\mathbf{m}}^{*, 1}\langle i-1\rangle} \beta_{i-1}^{J}+\sum_{\tilde{J} \in \mathcal{I}_{\mathbf{m}}^{*, 2}\langle i-1\rangle} \beta_{i-1}^{\tilde{J}},
$$

where $\beta_{i-1}^{J}=\prod_{\alpha=1}^{i}\left(\begin{array}{c}j_{\alpha+1} \\ j_{\alpha}\end{array}\right)$ and $\beta_{i-1}^{\tilde{J}}=\left[\prod_{\alpha=1}^{t}\left(\begin{array}{c}l_{\alpha+1} \\ l_{\alpha}\end{array}\right)\right]\left(\begin{array}{c}l_{t}-l_{t-1}-1 \\ k-l_{t-1}\end{array}\right)$ for $J=\left\{j_{1}, \ldots, j_{i}\right\} \in$ $\mathcal{I}_{\mathbf{m}}^{* 1}\langle i-1\rangle$ and $\tilde{J}=\left\{l_{1}, \ldots, l_{t}\right\} \in \mathcal{I}_{\mathbf{m}}^{*, 2}\langle i-1\rangle$. Here, $j_{i+1}=l_{t+1}=n$ and $l_{0}=0$.

Proof. Since $\mathcal{M}_{K_{n+1}}^{(k)}=I(\mathbf{u}(\mathbf{m}))^{[\mathbf{n}]}$, theorem follows from Theorem 3.2 and Corollary 3.4 of $[8]$.

Theorem 12 describes all multigraded Betti numbers of $\mathcal{M}_{K_{n+1}}^{(k)}$. We hope that it could be helpful in constructing a concrete minimal resolution of $\mathcal{M}_{K_{n+1}}^{(k)}$.

Corollary 13. Assume that $n \geqslant 3$ and $1 \leqslant i \leqslant n$. Then $\beta_{i-1}\left(\mathcal{M}_{K_{n+1}}^{(1)}\right)=i\left(\begin{array}{c}n+1 \\ i+1\end{array}\right)$ and

$$
\beta_{i-1}\left(\mathcal{M}_{K_{n+1}}^{(n-2)}\right)=\sum_{\mathbf{j}} \frac{n !}{j_{1} !\left(j_{2}-j_{1}\right) ! \cdots\left(n-j_{i}\right) !}+\sum_{\ell} \frac{n !\left(n-l_{i-2}-1\right)}{l_{1} !\left(l_{2}-l_{1}\right) ! \cdots\left(n-l_{i-2}\right) !}
$$

where the first and second summations run over all sequences of integers $\mathbf{j}=\left(j_{1}, \ldots, j_{i}\right)$ with $0<j_{1}<\cdots<j_{i}<n$ and $\ell=\left(l_{0}, l_{1}, \ldots, l_{i-2}\right)$ with $0=l_{0}<l_{1}<\cdots<l_{i-2}<n-1$, respectively.

Proof. For $k=1$, we have $\mathbf{m}=(1, n-1) \in \mathbb{N}^{2}$. We can easily see that $\mathcal{I}_{\mathbf{m}}^{*}\langle i-1\rangle=$ $\{\{1, i\},\{i+1\}\}$ for $i \geqslant 2$ and $\mathcal{I}_{\mathbf{m}}^{*}\langle 0\rangle=\{\{1\},\{2\}\}$. Thus, $\beta_{0}\left(\mathcal{M}_{K_{n+1}}^{(1)}\right)=\beta_{0}^{\{1\}}+\beta_{0}^{\{2\}}=$ $\left(\begin{array}{l}n \\ 1\end{array}\right)+\left(\begin{array}{l}n \\ 2\end{array}\right)=\left(\begin{array}{c}n+1 \\ 2\end{array}\right)$. For $i \geqslant 2$,

$$
\begin{aligned}
\beta_{i-1}\left(\mathcal{M}_{K_{n+1}}^{(1)}\right) & =\beta_{i-1}^{\{1, i\}}+\beta_{i-1}^{\{i+1\}}=\left(\begin{array}{l}
i \\
1
\end{array}\right)\left(\begin{array}{c}
n \\
i
\end{array}\right)\left(\begin{array}{c}
i-2 \\
0
\end{array}\right)+\left(\begin{array}{c}
n \\
i+1
\end{array}\right)\left(\begin{array}{l}
i \\
1
\end{array}\right) \\
& =i\left(\begin{array}{c}
n \\
i
\end{array}\right)+i\left(\begin{array}{c}
n \\
i+1
\end{array}\right)=i\left(\begin{array}{c}
n+1 \\
i+1
\end{array}\right),
\end{aligned}
$$

which is same as $\beta_{i}\left(\frac{R}{\mathcal{M}_{K_{n+1}}^{(1)}}\right)=\sum_{j=1}^{n} j\left(\begin{array}{c}j-1 \\ i-1\end{array}\right)=\sum_{j=1}^{n} i\left(\begin{array}{l}j \\ i\end{array}\right)=(i) \sum_{j=1}^{n}\left(\begin{array}{c}j \\ i\end{array}\right)=i\left(\begin{array}{c}n+1 \\ i+1\end{array}\right)$ obtained in [3]. 
For $k=n-2, J=\left\{j_{1}, \ldots, j_{i}\right\} \in \mathcal{I}_{\mathbf{m}}^{* 1}\langle i-1\rangle$ if and only if $J \subseteq[n-1]$ and $\beta_{i-1}^{J}=$ $\prod_{\alpha=1}^{i}\left(\begin{array}{c}j_{\alpha+1} \\ j_{\alpha}\end{array}\right)$. Also, $\tilde{J}=\left\{l_{1}, \ldots, l_{t}\right\} \in \mathcal{I}_{\mathbf{m}}^{*, 2}\langle i-1\rangle$ if and only if $l_{t-1} \leqslant n-2, l_{t}=n$ and $t=i-1$. Since, $\beta_{i-1}^{\tilde{J}}=\left[\prod_{\alpha=1}^{i-2}\left(\begin{array}{c}l_{\alpha+1} \\ l_{\alpha}\end{array}\right)\right]\left(\begin{array}{c}n-l_{i-2}-1 \\ n-l_{i-2}-2\end{array}\right)$, we get the desired expression for $\beta_{i-1}\left(\mathcal{M}_{K_{n+1}}^{(n-2)}\right)$.

Consider the first barycentric subdivision $\mathbf{B d}\left(\Delta_{n-1}\right)$ of an $n-1$-simplex $\Delta_{n-1}$. We construct a polyhedral cell complex $\mathbf{B d}^{(k)}\left(\Delta_{n-1}\right)$ whose vertices are the vertices of $\mathbf{B d}\left(\Delta_{n-1}\right)$ corresponding to subsets $A \subseteq[n]$ with $|A| \leqslant k+1$. An edge in $\mathbf{B d}^{(k)}\left(\Delta_{n-1}\right)$ corresponds either to a chain $A_{1} \subsetneq A_{2} \subseteq[n]$ with $\left|A_{2}\right| \leqslant k+1$ or a pair $\{A, B\}$ of subsets of $[n]$ with $|A|=|B|=k+1$ and $|A \backslash B|=1$. The higher dimensional faces of $\mathbf{B d}^{(k)}\left(\Delta_{n-1}\right)$ are polytopes spanned by its edges. A vertex of $\mathbf{B d}^{(k)}\left(\Delta_{n-1}\right)$ corresponding to $A$ with $|A| \leqslant k+1$ has a natural label $\left(\prod_{j \in A} x_{j}\right)^{n-|A|+1}$. The cellular resolution supported on the polyhedral cell complex $\mathbf{B d}^{(k)}\left(\Delta_{n-1}\right)$ is a non-minimal resolution of $\mathcal{M}_{K_{n+1}}^{(k)}$ if $1 \leqslant k \leqslant n-2$. The minimal cellular resolution of $\mathcal{M}_{K_{4+1}}^{(1)}$ constructed in [3] can be obtained by deleting certain edges of the polyhedral cell complex $\mathbf{B d}^{(1)}\left(\Delta_{3}\right)$.

\subsection{Standard monomials of $\mathcal{M}_{K_{n+1}}^{(k)}$}

A monomial $\mathbf{x}^{\mathbf{b}}=\prod_{j=1}^{n} x_{j}^{b_{j}} \notin \mathcal{M}_{K_{n+1}}^{(k)}$ is called a standard monomial of $\frac{R}{\mathcal{M}_{K_{n+1}}^{(k)}}$ or $\mathcal{M}_{K_{n+1}}^{(k)}$. Let $\lambda=\left(\lambda_{1}, \ldots, \lambda_{n}\right)$, where $\lambda_{i}=n-i+1$ for $1 \leqslant i \leqslant k$ and $\lambda_{j}=n-k$ for $k+1 \leqslant j \leqslant n$. We have seen that $I(\mathbf{u}(\mathbf{m}))^{[\mathbf{n}]}=\mathcal{M}_{K_{n+1}}^{(k)}=\mathcal{M}_{\lambda}$. In view of Theorem 4, the number of standard monomials of $\mathcal{M}_{K_{n+1}}^{(k)}$ is precisely the number of $\lambda$-parking functions and $\operatorname{dim}_{\mathbb{K}}\left(\frac{R}{\mathcal{M}_{K_{n+1}}^{(k)}}\right)=|\mathrm{PF}(\lambda)|=n ! \operatorname{det}(\Lambda(n, n-1, \ldots, n-k+1, n-k, \ldots, n-k))$.

More generally, for $a, b \geqslant 1$, we consider the complete multigraph $K_{n+1}^{a, b}$ on the vertex set $V$ with adjacency matrix $A\left(K_{n+1}^{a, b}\right)=\left[a_{i j}\right]_{0 \leqslant i, j \leqslant n}$ given by $a_{0, i}=a_{i, 0}=a$ and $a_{i, j}=b$ for $i, j \in V \backslash\{0\} ; i \neq j$. In other words, $K_{n+1}^{a, b}$ has exactly a number of edges between the root 0 and any other vertex $i$, while it has exactly $b$ number of edges between distinct non-root vertices $i$ and $j$. Clearly, $K_{n+1}^{1,1}=K_{n+1}$. The $k$-skeleton ideal $\mathcal{M}_{K_{n+1}^{a, b}}^{(k)}$ of $K_{n+1}^{a, b}$ is given by

$$
\mathcal{M}_{K_{n+1}^{a, b}}^{(k)}=\left\langle\left(\prod_{j \in A} x_{j}\right)^{a+(n-|A|) b}: \emptyset \neq A \subseteq[n] ;|A| \leqslant k+1\right\rangle .
$$

Let $\lambda^{a, b}=\left(\lambda_{1}^{a, b}, \ldots, \lambda_{n}^{a, b}\right)$, where $\lambda_{i}^{a, b}=a+(n-i) b$ for $1 \leqslant i \leqslant k$ and $\lambda_{j}^{a, b}=a+(n-k-1) b$ for $k+1 \leqslant j \leqslant n$. Then, $\mathcal{M}_{K_{n+1}^{a, b}}^{(k)}=\mathcal{M}_{\lambda^{a, b}}$ and from Theorem 4 ,

$$
\operatorname{dim}_{\mathbb{K}}\left(\frac{R}{\mathcal{M}_{K_{n+1}^{a, b}}^{(k)}}\right)=n ! \operatorname{det}\left(\Lambda\left(\lambda_{1}^{a, b}, \ldots, \lambda_{n}^{a, b}\right)\right) .
$$


We proceed to evaluate the Steck determinant and compute the number of standard monomials of $\mathcal{M}_{K_{n+1}^{a, b}}^{(k)}$. Consider the polynomial

$$
f_{n}(x)=\operatorname{det}(\Lambda(x+(n-1) b, x+(n-2) b, \ldots, x+b, x))
$$

in an indeterminate $x$. In other words, we have

$$
f_{n}(x)=\operatorname{det}\left[\begin{array}{cccccc}
\frac{x}{1 !} & \frac{x^{2}}{2 !} & \frac{x^{3}}{3 !} & \cdots & \frac{x^{n-1}}{(n-1) !} & \frac{x^{n}}{n !} \\
1 & \frac{x+b}{1 !} & \frac{(x+b)^{2}}{2 !} & \cdots & \frac{(x+b)^{n-2}}{(n-2) !} & \frac{(x+b)^{n-1}}{(n-1) !} \\
0 & 1 & \frac{x+2 b}{1 !} & \cdots & \frac{(x+2 b)^{n-3}}{(n-3) !} & \frac{(x+2 b)^{n-2}}{(n-2) !} \\
\vdots & \vdots & \vdots & \ddots & \vdots & \vdots \\
0 & 0 & 0 & \cdots & \frac{(x+(n-2) b)}{1 !} & \frac{(x+(n-2) b)^{2}}{2 !} \\
0 & 0 & 0 & \cdots & 1 & \frac{x+(n-1) b}{1 !}
\end{array}\right] .
$$

The polynomial $f_{n}(x)=\frac{x(x+n b)^{n-1}}{n !}$ and $\operatorname{dim}_{\mathbb{K}}\left(\frac{R}{\mathcal{M}_{K_{n+1}^{a, b}}}\right)=a(a+n b)^{n-1}$ (see $\left.[14,15]\right)$. Also, for $1 \leqslant k \leqslant n-2$, consider another polynomial $g_{n ; k}(x)$ in $x$ given by

$$
g_{n ; k}(x)=\operatorname{det}(\Lambda(x+k b, x+(k-1) b, \ldots, x+b, x, \ldots, x)),
$$

where the last $n-k$ coordinates in $(x+k b, x+(k-1) b, \ldots, x+b, x, \ldots, x)$ are $x$.

Proposition 14. The polynomial $g_{n ; k}(x)$ is given by

$$
g_{n ; k}(x)=\sum_{j=0}^{k} \frac{1}{j !} \frac{x^{n-j}}{(n-j) !}(k-j+1)(k+1)^{j-1} b^{j} .
$$

Proof. We first give a simple proof of $f_{n}(x)=\frac{x(x+n b)^{n-1}}{n !}$ as in [9]. Clearly, $f_{1}(x)=x$ and $f_{2}(x)=\frac{x(x+2 b)}{2 !}$. Proceeding by induction on $n$, we assume that $f_{j}(x)=\frac{x(x+j b)^{j-1}}{j !}$ for $1 \leqslant j \leqslant n-1$. Further, using properties of determinants, we observe that the derivative $f_{n}^{\prime}(x)$ of $f_{n}(x)$ satisfies $f_{n}^{\prime}(x)=f_{n-1}(x+b)$. This shows that $f_{n}^{\prime}(x)=\frac{(x+b)(x+n b)^{n-2}}{(n-1) !}$. As $f_{n}(0)=0$, on integrating $f_{n}^{\prime}(x)=\frac{(x+b)(x+n b)^{n-2}}{(n-1) !}$ by parts, we get $f_{n}(x)=\frac{x(x+n b)^{n-1}}{n !}$.

Again using properties of determinants, we see that the $(n-k-1)^{t h}$ derivative $g_{n ; k}^{(n-k-1)}(x)$ of $g_{n ; k}(x)$ satisfies

$$
g_{n ; k}^{(n-k-1)}(x)=f_{k+1}(x)=\frac{x(x+(k+1) b)^{k}}{(k+1) !}=\sum_{j=0}^{k}\left(\begin{array}{c}
k \\
j
\end{array}\right) x^{k-j+1} \frac{(k+1)^{j} b^{j}}{(k+1) !} .
$$

Since $g_{n ; k}(0)=g_{n ; k}^{\prime}(0)=\cdots=g_{n ; k}^{(n-k-1)}(0)=0$ and the $(n-k-1)^{t h}$ derivative of $\frac{x^{n-j}}{(n-j)(n-j-1) \ldots(k-j+2)}$ is $x^{k-j+1}$, we get $g_{n ; k}(x)=\sum_{j=0}^{k}\left(\begin{array}{c}k \\ j\end{array}\right) \frac{x^{n-j}}{(n-j)(n-j-1) \cdots(k-j+2)} \frac{(k+1)^{j} b^{j}}{(k+1) !}$. 
Theorem 15 (Yan). The number of standard monomials of $\frac{R}{\mathcal{M}_{K_{n+1}^{(k)}}^{a, b}}$ is given by

$$
\operatorname{dim}_{\mathbb{K}}\left(\frac{R}{\mathcal{M}_{K_{n+1}^{a, b}}^{(k)}}\right)=\sum_{j=0}^{k}\left(\begin{array}{l}
n \\
j
\end{array}\right)(a+(n-k-1) b)^{n-j}(k-j+1)(k+1)^{j-1} b^{j} .
$$

In particular, we have $\operatorname{dim}_{\mathbb{K}}\left(\frac{R}{\mathcal{M}_{K_{n+1}^{(1)}}^{a, b}}\right)=(a+(n-2) b)^{n-1}(a+(2 n-2) b)$ for $k=1$ and $\operatorname{dim}_{\mathbb{K}}\left(\frac{R}{\mathcal{M}_{K_{n+1}^{(n-2)}}^{a, b}}\right)=a(a+n b)^{n-1}+(n-1)^{n-1} b^{n}$ for $k=n-2$.

Proof. The first part follows from $\operatorname{dim}_{\mathbb{K}}\left(\frac{R}{\mathcal{M}_{K_{n+1}^{(k)}}^{a, b}}\right)=n ! g_{n ; k}(a+(n-k-1) b)$ using Proposition 14.

For $k=1, g_{n ; 1}^{(n-2)}(x)=f_{2}(x)=\frac{x(x+2 b)}{2 !}=\frac{x^{2}}{2 !}+b x$. As $g_{n ; 1}^{(j)}(0)=0$ for $0 \leqslant j \leqslant n-2$, we obtain

$$
g_{n ; 1}(x)=\frac{x^{n}}{n !}+\frac{b x^{n-1}}{(n-1) !}=\frac{x^{n-1}(x+n b)}{n !} .
$$

Now $\operatorname{dim}_{\mathbb{K}}\left(\frac{R}{\mathcal{M}_{K_{n+1}^{(1)}}^{(1)}}\right)=n ! g_{n ; 1}(a+(n-2) b)=(a+(n-2) b)^{n-1}(a+(2 n-2) b)$.

Also, for $k=n-2$, we have $g_{n ; n-2}^{\prime}(x)=f_{n-1}(x)=\frac{x(x+(n-1) b)^{n-2}}{(n-1) !}$. On integrating it by parts, we get $g_{n ; n-2}(x)=\frac{x(x+(n-1) b)^{n-1}}{(n-1) !(n-1)}-\frac{(x+(n-1) b)^{n}}{n !(n-1)}+C$, where $C$ is a constant of integration. Since $g_{n ; n-2}(0)=0$, we get $C=\frac{(n-1)^{n-1} b^{n}}{n !}$. Hence,

$$
g_{n ; n-2}(x)=\frac{1}{n !}\left[(x-b)(x+(n-1) b)^{n-1}+(n-1)^{n-1} b^{n}\right] .
$$

Again, from $\operatorname{dim}_{\mathbb{K}}\left(\frac{R}{\mathcal{M}_{K_{n+1}^{(n-2)}}^{a, b}}\right)=n ! g_{n ; n-2}(a+b)$, we get the desired result.

Remark 16. The determinant $\operatorname{det}\left(Q_{K_{n+1}^{a, b}}\right)$ of the reduced signless Laplacian matrix $Q_{K_{n+1}^{a, b}}$ of $K_{n+1}^{a, b}$ satisfies $\operatorname{dim}_{\mathbb{K}}\left(\frac{R}{\mathcal{M}_{K_{n+1}^{(1)}}^{a, b}}\right)=(a+(n-2) b)^{n-1}(a+(2 n-2) b)=\operatorname{det}\left(Q_{K_{n+1}^{a, b}}\right)$. Also, we have $g_{n ; n-2}^{\prime}(x)=f_{n-1}(x)=\frac{x(x+(n-1) b)^{n-2}}{(n-1) !}=\sum_{j=0}^{n-2}\left(\begin{array}{c}n-2 \\ j\end{array}\right) \frac{x^{n-1-j}}{(n-1) !}(n-1)^{j} b^{j}$. Thus on integrating $g_{n ; n-2}^{\prime}(x)$ in two ways, we get $g_{n ; n-2}(x)$ and a polynomial identity

$$
\frac{(x-b)(x+(n-1) b)^{n-1}+(n-1)^{n-1} b^{n}}{n !}=\frac{\sum_{j=0}^{n-2}\left(\begin{array}{l}
n \\
j
\end{array}\right) x^{n-j}(n-j-1)(n-1)^{j-1} b^{j}}{n !} .
$$


On substituting $x=a+b$, we get an identity

$$
\sum_{j=0}^{n-2}\left(\begin{array}{l}
n \\
j
\end{array}\right)(a+b)^{n-j}(n-j-1)(n-1)^{j-1} b^{j}=a(a+n b)^{n-1}+(n-1)^{n-1} b^{n}
$$

for positive integers $a$ and $b$. Taking $a=b=1$, it justifies the equality

$$
\sum_{j=0}^{n-2}\left(\begin{array}{l}
n \\
j
\end{array}\right) 2^{n-j}(n-j-1)(n-1)^{j-1}=(n+1)^{n-1}+(n-1)^{n-1}
$$

described in [4](Corollary 3.7).

\section{Spherical $G$-parking functions}

Let $G$ be a connected graph on the vertex set $V=\{0,1, \ldots, n\}$ with root 0 . As stated in the Introduction, $\mathcal{P}:[n] \rightarrow \mathbb{N}$ is a spherical $G$-parking function if $\mathbf{x}^{\mathcal{P}}=\prod_{i \in[n]} x_{i}^{\mathcal{P}(i)} \in$ $\mathcal{M}_{G} \backslash \mathcal{M}_{G}^{(n-2)}$. Let $\operatorname{PF}(G)$ (or $\operatorname{sPF}(G)$ ) be the set of $G$-parking functions (respectively, spherical $G$-parking functions).

Let $e_{0}$ be an edge of $G$ joining the root 0 to another vertex. We shall compare $\operatorname{sPF}(G)$ with $\operatorname{sPF}(\bar{G})$, where $\bar{G}=G-\left\{e_{0}\right\}$. After renumbering vertices, we may assume that $e_{0}=e_{0, n}$ is an edge joining the root 0 with $n$.

Lemma 17. Let $G$ be a connected graph on the vertex set $V$ and $\bar{G}=G-\left\{e_{0}\right\}$. Then

$$
\mathcal{M}_{\bar{G}}=\left(\mathcal{M}_{G}: x_{n}\right)=\left\{z \in R: z x_{n} \in \mathcal{M}_{G}\right\} .
$$

Further, the multiplication map $\mu_{x_{n}}:\left\{\mathbf{x}^{\mathcal{P}}: \mathcal{P} \in \operatorname{sPF}(\bar{G})\right\} \rightarrow\left\{\mathbf{x}^{\mathcal{P}}: \mathcal{P} \in \operatorname{sPF}(G)\right\}$ induced by $x_{n}$ is a bijection. In particular, $|\operatorname{sPF}(G)|=|\operatorname{sPF}(\bar{G})|$.

Proof. For $\emptyset \neq A \subseteq[n]$, let $m_{A}$ and $m_{A}^{\prime}$ be the generators of $\mathcal{M}_{G}$ and $\mathcal{M}_{\bar{G}}$, respectively. Clearly, $m_{A}=m_{A}^{\prime}$ if $n \notin A$ and $m_{A}=m_{A}^{\prime} x_{n}$ if $n \in A$. This shows that $\mathcal{M}_{\bar{G}}=\left(\mathcal{M}_{G}: x_{n}\right)$. Also, $\mathcal{M}_{\bar{G}}^{(n-2)}=\left(\mathcal{M}_{G}^{(n-2)}: x_{n}\right)$. Thus the natural sequences of $R$-modules (or $\mathbb{K}$-vectors spaces)

$$
0 \rightarrow \frac{R}{\mathcal{M}_{\bar{G}}} \stackrel{\mu_{x_{n}}}{\rightarrow} \frac{R}{\mathcal{M}_{G}} \rightarrow \frac{R}{\left\langle\mathcal{M}_{G}, x_{n}\right\rangle} \rightarrow 0 \text { and } 0 \rightarrow \frac{R}{\mathcal{M}_{\bar{G}}^{(n-2)}} \stackrel{\mu_{x_{n}}}{\rightarrow} \frac{R}{\mathcal{M}_{G}^{(n-2)}} \rightarrow \frac{R}{\left\langle\mathcal{M}_{G}^{(n-2)}, x_{n}\right\rangle} \rightarrow 0
$$

are short exact. Let $\alpha: \frac{R}{\mathcal{M}_{\bar{G}}^{(n-2)}} \rightarrow \frac{R}{\mathcal{M}_{\bar{G}}}$ and $\beta: \frac{R}{\mathcal{M}_{G}^{(n-2)}} \rightarrow \frac{R}{\mathcal{M}_{G}}$ be the natural projections. Since $\left\langle\mathcal{M}_{G}, x_{n}\right\rangle=\left\langle\mathcal{M}_{G}^{(n-2)}, x_{n}\right\rangle$, the multiplication map $\mu_{x_{n}}$ induces an isomorphism $\operatorname{ker}(\alpha) \stackrel{\sim}{\rightarrow} \operatorname{ker}(\beta)$ between kernels $\operatorname{ker}(\alpha)$ and $\operatorname{ker}(\beta) . \quad$ Also $\left\{\mathbf{x}^{\mathcal{P}}: \mathcal{P} \in \operatorname{sPF}(\bar{G})\right\}$ and $\left\{\mathbf{x}^{\mathcal{P}}: \mathcal{P} \in \operatorname{sPF}(G)\right\}$ are monomial basis of $\operatorname{ker}(\alpha)$ and $\operatorname{ker}(\beta)$, respectively. Thus $\mu_{x_{n}}$ induces a bijection between the bases.

We now give a few applications of the Lemma 17. 
Proposition 18. Let $E$ be the set of all edges of $K_{n+1}$ or $K_{n+1}^{a, b}$ through the root 0 . Then

(1) $\left|\operatorname{sPF}\left(K_{n+1}-E\right)\right|=\left|\operatorname{sPF}\left(K_{n+1}\right)\right|$.

(2) $\left|\operatorname{sPF}\left(K_{n+1}^{a, b}-E\right)\right|=\left|\operatorname{sPF}\left(K_{n+1}^{a, b}\right)\right|$.

(3) $\left|\operatorname{sPF}\left(K_{n+1}^{a, b}\right)\right|=b^{n}(n-1)^{n-1}$.

Proof. By Lemma 17, we know that the number of spherical $G$-parking functions and the number of spherical $\left(G-\left\{e_{0}\right\}\right)$-parking functions are the same for any edge $e_{0}$ of $G$ through the root 0 . Now, repeatedly applying Lemma 17, we see that (1) and (2) hold.

Let $\lambda=((n-1) b,(n-2) b, \ldots, 2 b, b, b)$. Consider the graph $K_{n+1}^{a, b}-E$ and its $(n-$ 2)-skeleton ideal $\mathcal{M}_{K_{n+1}^{a, b}-E}^{(n-2)}$. Clearly, $\mathcal{M}_{K_{n+1}^{a, b}-E}^{(n-2)}=\mathcal{M}_{\lambda}$. As $K_{n+1}^{a, b}-E$ is disconnected, $\operatorname{PF}\left(K_{n+1}^{a, b}-E\right)=\emptyset$. Thus

$$
\begin{aligned}
\left|\operatorname{sPF}\left(K_{n+1}^{a, b}\right)\right| & =\left|\operatorname{sPF}\left(K_{n+1}^{a, b}-E\right)\right|=\operatorname{dim}_{\mathbb{K}}\left(\frac{R}{\mathcal{M}_{K_{n+1}^{(n-2)}-E}^{a, b}}\right) \\
& =|\mathrm{PF}(\lambda)|=(n !) g_{n ; n-2}(b)=b^{n}(n-1)^{n-1},
\end{aligned}
$$

where the polynomial $g_{n ; n-2}(x)$ is given in the Remark 16 .

Note that the cardinality $\left|\operatorname{sPF}\left(K_{n+1}^{a, b}\right)\right|$ is independent of $a$. As we have seen that $\left|\mathrm{PF}\left(K_{n+1}^{a, b}\right)\right|=a(a+b n)^{n-1},\left|\operatorname{sPF}\left(K_{n+1}^{a, b}\right)\right|=b^{n}(n-1)^{n-1}$ also follows from Theorem 15.

\subsection{A modified Depth-First-Search burning algorithm}

Let $G$ be a connected simple graph on the vertex set $V$ with a root 0 . Let $\mathcal{M}_{G}=\left\langle m_{A}: \emptyset \neq\right.$ $A \subseteq[n]\rangle$ be the $G$-parking function ideal. For a spherical $G$-parking function $\mathcal{P} \in \operatorname{sPF}(G)$, define $\widetilde{\mathcal{P}}:[n] \rightarrow \mathbb{N}$ so that $\mathbf{x}^{\widetilde{\mathcal{P}}}=\frac{\mathbf{x}^{\mathcal{P}}}{m_{[n]}}$, where $m_{[n]}$ is the generator of $\mathcal{M}_{G}$ corresponding to $[n]$. We say that $\widetilde{\mathcal{P}}$ is the reduced spherical $G$-parking function associated to $\mathcal{P} \in \operatorname{sPF}(G)$. Let $\widetilde{\operatorname{sPF}}(G)=\{\widetilde{\mathcal{P}}: \mathcal{P} \in \operatorname{sPF}(G)\}$ be the set of reduced spherical $G$-parking functions. We shall analyse the condition $\widehat{\operatorname{sPF}}(G) \subseteq \operatorname{PF}(G)$. Since removing (or adding) edges from the root 0 to another vertex in $G$ do not change the number of spherical $G$-parking functions (Lemma 17), we may assume that the root 0 is connected to all the other vertices in $G$. In this case, $m_{[n]}=x_{1} x_{2} \cdots x_{n}$ and $\widetilde{\mathcal{P}}(i)=\mathcal{P}(i)-1$ for $i \in[n]$.

Lemma 19. Let $G$ be a connected simple graph on the vertex set $V$ with a root 0 . Suppose the root 0 is connected to all other vertices of $G$. Then

(i) $\widetilde{\operatorname{sPF}}(G) \subseteq \operatorname{PF}(G)$.

(ii) Let $\mathcal{P} \in \operatorname{sPF}(G)$ and $r \in[n]$ be the unique vertex such that $\widetilde{\mathcal{P}}(r)=0$ but $\widetilde{\mathcal{P}}(j) \geqslant 1$ for $j \geq r$. Consider the graph $G^{\prime}=G-\{0\}$ on the vertex set $[n]$ with root $r$. Then $\widehat{\mathcal{P}}=\left.\widetilde{\mathcal{P}}\right|_{[n] \backslash\{r\}}$ is a $G^{\prime}$-parking function. 
Proof. Let $\mathcal{P} \in \operatorname{sPF}(G)$ such that $\widetilde{\mathcal{P}} \notin \operatorname{PF}(G)$. Then there exists $\emptyset \neq A \subseteq[n]$ such that $m_{A} \mid \mathbf{x}^{\widetilde{\mathcal{P}}}$, i.e., $m_{A}$ divides $\mathbf{x}^{\widetilde{\mathcal{P}}}$. Thus $m_{A} m_{[n]} \mid \mathbf{x}^{\mathcal{P}}$. If $A \neq[n]$, then $m_{A} \mid \mathbf{x}^{\mathcal{P}}$, a contradiction to $\mathcal{P} \in \operatorname{sPF}(G)$. Also, if $A=[n]$, then $\left(m_{[n]}\right)^{2} \mid \mathbf{x}^{\mathcal{P}}$. Since $G$ is a simple graph and the root 0 is connected to all other vertices of $G, m_{B} \mid\left(x_{1} x_{2} \cdots x_{n}\right)^{2}$ for any $B \subseteq[n]$ with $|B|=n-1$. Again a contradiction. This proves the first part.

Let $\mathcal{P} \in \operatorname{sPF}(G)$. If $\widetilde{\mathcal{P}}(i) \geqslant 1$ for all $i \in[n]$, then $\mathcal{P}(i) \geqslant 2$ for all $i$. Thus $\left(m_{[n]}\right)^{2} \mid \mathbf{x}^{\mathcal{P}}$, which leads to a contradiction. Thus $\widetilde{\mathcal{P}}(i)=0$ for some $i$. Let $r=\max \{i \in[n]: \widetilde{\mathcal{P}}(i)=0\}$. Now consider the graph $G^{\prime}=G-\{0\}$ on the vertex set $[n]$ with root $r$. When we emphasize the root $r$ of $G^{\prime}$, we denote this graph by $\left(G^{\prime}, r\right)$. Let $\mathcal{M}_{\left(G^{\prime}, r\right)}=\left\langle\bar{m}_{A}: \emptyset \neq A \subseteq[n] \backslash\{r\}\right\rangle$ be the $G^{\prime}$-parking function ideal in the polynomial ring $\mathbb{K}\left[x_{1}, \ldots, \widehat{x_{r}}, \ldots, x_{n}\right]$. We see that $\bar{m}_{A}=\frac{m_{A}}{\operatorname{gcd}\left(m_{A}, m_{[n]}\right)}$. If $\widehat{\mathcal{P}}=\left.\widetilde{\mathcal{P}}\right|_{[n] \backslash\{r\}}$ is not a $G^{\prime}$-parking function, then $\bar{m}_{A} \mid \prod_{i \in[n] \backslash\{r\}}\left(x_{i}\right)^{\widehat{\mathcal{P}}(i)}$ for some non-empty subset $A \subseteq[n] \backslash\{r\}$. As $\widetilde{\mathcal{P}}(r)=0, \mathbf{x}^{\widetilde{\mathcal{P}}}=\prod_{i \in[n] \backslash\{r\}}\left(x_{i}\right)^{\widehat{\mathcal{P}}(i)}=\frac{\mathbf{x}^{\mathcal{P}}}{m_{[n]}}$. Thus $m_{A} \mid \mathbf{x}^{\mathcal{P}}$, a contradiction to $\mathcal{P} \in \operatorname{sPF}(G)$.

We now proceed to associate uprooted trees to spherical parking functions by modifying the Depth-First-Search burning algorithm. Let $G$ be a connected simple graph satisfying the hypothesis of Lemma 19 . Let $\mathcal{P} \in \operatorname{sPF}(G)$ and $\widetilde{\mathcal{P}}$ be the associated reduced spherical $G$-parking function. In the following three steps, an uprooted spanning tree of $G^{\prime}$ is associated to each $\mathcal{P} \in \operatorname{sPF}(G)$.

1. Set $r=\max \{i \in[n]: \widetilde{\mathcal{P}}(i)=0\}$ and consider the graph $G^{\prime}=G-\{0\}$ with root $r$.

2. Let $\phi: \operatorname{PF}\left(G^{\prime}, r\right) \rightarrow \operatorname{SPT}\left(G^{\prime}, r\right)$ be the bijective map induced by Depth-First-Search algorithm (Theorem 8). As $\widehat{\mathcal{P}}=\left.\widetilde{\mathcal{P}}\right|_{[n] \backslash\{r\}}$ is a $\left(G^{\prime}, r\right)$-parking function, $\phi(\widehat{\mathcal{P}})$ is a spanning tree of $G^{\prime}$. Also, $\operatorname{sum}(\widehat{\mathcal{P}})=g\left(G^{\prime}\right)-\kappa\left(G^{\prime}, \phi(\widehat{\mathcal{P}})\right)$.

3. Since $\widehat{\mathcal{P}} \in \operatorname{PF}\left(G^{\prime}, r\right)$ and $\widehat{\mathcal{P}}(j) \geqslant 1$ for all $j>r$, there exists $i<r$ such that $\widehat{\mathcal{P}}(i)=0$. On applying the Depth-First-Search algorithm to $\widehat{\mathcal{P}}$, all the edges $(r, j)$ for $j>r$ get dampened. Thus the spanning tree $\phi(\widehat{\mathcal{P}})$ is an uprooted spanning tree of $G^{\prime}$.

Let $\mathcal{U}\left(G^{\prime}\right)$ be the set of uprooted spanning trees of the graph $G^{\prime}$. We define a map $\phi_{G}: \operatorname{sPF}(G) \rightarrow \mathcal{U}\left(G^{\prime}\right)$ given by $\phi_{G}(\mathcal{P})=\phi(\widehat{\mathcal{P}})$, where $\widehat{\mathcal{P}}=\left.\widetilde{\mathcal{P}}\right|_{[n] \backslash\{r\}}$. We say that the map $\phi_{G}$ is induced by a modified Depth-First-Search algorithm.

Theorem 20. Let $G$ be a simple graph on the vertex set $V$ with root 0 and $G^{\prime}=G-\{0\}$. Suppose the root 0 is connected to all other vertices of $G$. Then there exists an injective map $\phi_{G}: \operatorname{sPF}(G) \rightarrow \mathcal{U}\left(G^{\prime}\right)$ such that $\operatorname{sum}(\mathcal{P})=g(G)-\kappa\left(G^{\prime}, \phi_{G}(\mathcal{P})\right)+1 \quad$ for all $\mathcal{P} \in \operatorname{sPF}(G)$.

Proof. We have already constructed the map $\phi_{G}$. Let $\mathcal{P}, \mathcal{P}^{\prime} \in \operatorname{sPF}(G)$ such that $\phi_{G}(\mathcal{P})=$ $\phi_{G}\left(\mathcal{P}^{\prime}\right)=T \in \mathcal{U}\left(G^{\prime}\right)$. Let $r$ be the root of $T$. Since $\phi: \operatorname{PF}\left(G^{\prime}, r\right) \rightarrow \operatorname{SPT}\left(G^{\prime}, r\right)$ is a bijection and $\phi(\widehat{\mathcal{P}})=\phi\left(\widehat{\mathcal{P}^{\prime}}\right)$, we have $\widehat{\mathcal{P}}=\widehat{\mathcal{P}^{\prime}}$ and hence $\mathcal{P}=\mathcal{P}^{\prime}$. Note that $\operatorname{sum}(\mathcal{P})=$ $\operatorname{sum}(\widehat{\mathcal{P}})+n$ and $g(G)=g\left(G^{\prime}\right)+n-1$. Thus $\operatorname{sum}(\mathcal{P})=g(G)-\kappa\left(G^{\prime}, \phi_{G}(\mathcal{P})\right)+1$ follows from $\operatorname{sum}(\widehat{\mathcal{P}})=g\left(G^{\prime}\right)-\kappa\left(G^{\prime}, \phi(\widehat{\mathcal{P}})\right)$. 
Let $\operatorname{Im}\left(\phi_{G}\right)=\left\{\phi_{G}(\mathcal{P}): \mathcal{P} \in \operatorname{sPF}(G)\right\}$ be the image of $\phi_{G}$ in $\mathcal{U}\left(G^{\prime}\right)$. Theorem 20 shows that under some mild conditions on the simple graph $G$, the spherical $G$-parking functions correspond bijectively with the uprooted trees in $\operatorname{Im}\left(\phi_{G}\right)$. In general, it is not easy to give a combinatorial description for the image $\operatorname{Im}\left(\phi_{G}\right)$.

Let $T \in \mathcal{U}\left(G^{\prime}\right)$ be an uprooted spanning tree of $G^{\prime}=G-\{0\}$. Suppose $\operatorname{root}(T)=r$. Consider the bijective map $\phi: \operatorname{PF}\left(G^{\prime}, r\right) \rightarrow \operatorname{SPT}\left(G^{\prime}, r\right)$. Then there exists a unique $\left(G^{\prime}, r\right)$-parking function $\mathcal{P}_{T}$ such that $\phi\left(\mathcal{P}_{T}\right)=T$. Let

$$
\overline{\mathcal{U}}\left(G^{\prime}\right)=\left\{T \in \mathcal{U}\left(G^{\prime}\right): \mathcal{P}_{T}(j) \geqslant 1 \text { for } j>r=\operatorname{root}(T)\right\} .
$$

Proposition 21. $\operatorname{Im}\left(\phi_{G}\right) \subseteq \overline{\mathcal{U}}\left(G^{\prime}\right)=\left\{T \in \mathcal{U}\left(G^{\prime}\right): \mathcal{P}_{T}(j) \geqslant 1\right.$ for $\left.j>r=\operatorname{root}(T)\right\}$.

Proof. Let $\phi_{G}(\mathcal{P})=\phi(\widehat{\mathcal{P}})=T$, where $\widehat{\mathcal{P}}=\left.\widetilde{\mathcal{P}}\right|_{[n] \backslash\{r\}}$. As $\mathcal{P}_{T}=\widehat{\mathcal{P}}$ and the root is given by $\operatorname{root}(T)=\max \{i \in[n]: \widetilde{\mathcal{P}}(i)=0\}$, the result follows.

\subsection{Spherical parking functions for complete graphs}

Let $K_{n+1}$ be the complete graph on the vertex set $V$ and $K_{n}=K_{n+1}-\{0\}$ be the complete graph on the vertex set $[n]$. Let $\mathcal{U}_{n}=\mathcal{U}\left(K_{n}\right)$ be the set of uprooted trees on the vertex set $[n]$. From Theorem 20, there exists an injective map $\phi_{n}=\phi_{K_{n+1}}: \operatorname{sPF}\left(K_{n+1}\right) \rightarrow \mathcal{U}_{n}$. We show that $\phi_{n}$ is a bijection and solve a conjecture of Dochtermann on spherical $K_{n+1^{-}}$ parking functions.

Theorem 22. There exists a bijection $\phi_{n}: \operatorname{sPF}\left(K_{n+1}\right) \rightarrow \mathcal{U}_{n}$ such that

$$
\operatorname{sum}(\mathcal{P})=\left(\begin{array}{l}
n \\
2
\end{array}\right)-\kappa\left(K_{n}, \phi_{n}(\mathcal{P})\right)+1 \quad \text { for all } \quad \mathcal{P} \in \operatorname{sPF}\left(K_{n+1}\right)
$$

Proof. The existence of injective map $\phi_{n}=\phi_{K_{n+1}}: \operatorname{sPF}\left(K_{n+1}\right) \rightarrow \mathcal{U}_{n}$ with the desired property follows from the Theorem 20. We just need to show that $\phi_{n}$ is surjective. Let $T \in \mathcal{U}_{n}$ and $\operatorname{root}(T)=r$. Consider the bijective map $\phi: \operatorname{PF}\left(K_{n}, r\right) \rightarrow \operatorname{SPT}\left(K_{n}, r\right)$ induced by Depth-First-Search algorithm and $\mathcal{P}_{T}$ is the unique $\left(K_{n}, r\right)$-parking function such that $\phi\left(\mathcal{P}_{T}\right)=T$. Since $T$ is uprooted, $\mathcal{P}_{T}(j) \geqslant 1$ for $j>r$. Now consider ideals $\mathcal{M}_{K_{n+1}}=\left\langle m_{A}: \emptyset \neq A \subseteq[n]\right\rangle$ and $\mathcal{M}_{\left(K_{n}, r\right)}=\left\langle\bar{m}_{B}: \emptyset \neq B \subseteq[n] \backslash\{r\}\right\rangle$.

Suppose, if possible, $\mathcal{P}_{T} \neq \widehat{\mathcal{P}}$ for all $\mathcal{P} \in \operatorname{sPF}\left(K_{n+1}\right)$. Then $m_{[n]} \prod_{j \in[n] \backslash\{r\}} x_{j}^{\mathcal{P}_{T}^{(j)}}$ is not a standard monomial of $\mathcal{M}_{K_{n+1}}^{(n-2)}$. Thus there exists $\emptyset \neq A \subsetneq[n]$ such that $m_{A}$ divides $m_{[n]} \prod_{j \in[n] \backslash\{r\}} x_{j}^{\mathcal{P}_{T}^{(j)}}$. If $r \in A$, then $x_{r}$ appearing in $m_{A}=\left(\prod_{j \in A} x_{j}\right)^{n-|A|+1}$ must have the multiplicity 1. This is possible, only if $A=[n]$, a contradiction. If $r \notin A$, then $\bar{m}_{A}=\frac{m_{A}}{\operatorname{gcd}\left(m_{A}, m_{[n]}\right)}$ and $\bar{m}_{A} \mid \prod_{j \in[n] \backslash\{r\}} x_{j}^{\mathcal{P}_{T}(j)}$. This shows that $\mathcal{P}_{T}$ is not a $\left(K_{n}, r\right)$-parking function, again a contradiction. Hence $\phi_{n}$ is surjective.

The surjectivity of $\phi_{n}$ also follows from $\left|\operatorname{sPF}\left(K_{n+1}\right)\right|=\left|\mathcal{U}_{n}\right|=(n-1)^{n-1}$.

We now study spherical $G$-parking functions for $G=K_{n+1}-\{e\}$, where $e$ is an edge not through the root 0 . Let $e=e_{p, q}=(p, q)$ be the edge in $K_{n+1}$ joining $p$ and 
$q$ with $1 \leqslant p<q \leqslant n$. Let $G^{\prime}=G-\{0\}$ be the graph on the vertex set $[n]$ and $\mathcal{U}\left(G^{\prime}\right)$ be the set of uprooted spanning trees of $G^{\prime}$. In fact, $\mathcal{U}_{n}^{(p \nsim q)}=\mathcal{U}\left(G^{\prime}\right)$ is the set of uprooted trees on the vertex set $[n]$ with no edge between $p$ and $q$ (i.e., $p \nsim q$ ). Let $\overline{\mathcal{U}}_{n}^{(p \nsim q)}=\overline{\mathcal{U}}\left(G^{\prime}\right)=\left\{T \in \mathcal{U}\left(G^{\prime}\right): \mathcal{P}_{T}(j) \geqslant 1\right.$ for $\left.j>r=\operatorname{root}(T)\right\}$ as in Proposition 21 and set $\mathcal{U}_{n}^{\prime}=\mathcal{U}_{n}^{(1 \nsim n)}$. In view of Theorem 20 and Proposition 21, there exists an injective map $\phi_{G}: \operatorname{sPF}(G) \rightarrow \overline{\mathcal{U}}_{n}^{(p \nsim q)}$.

Theorem 23. For $n \geqslant 3$ and $G=K_{n+1}-\left\{e_{p, q}\right\}$, the map $\phi_{G}: \operatorname{sPF}(G) \rightarrow \overline{\mathcal{U}}_{n}^{(p \nsim q)}$ is a bijection such that $\operatorname{sum}(\mathcal{P})=\left(\begin{array}{l}n \\ 2\end{array}\right)-\kappa\left(G^{\prime}, \phi_{G}(\mathcal{P})\right)$ for all $\mathcal{P} \in \operatorname{sPF}(G)$, where $G^{\prime}=G-\{0\}$.

Proof. We only need to show that $\operatorname{Im}\left(\phi_{G}\right)=\overline{\mathcal{U}}\left(G^{\prime}\right)$. This proof is similar to the proof of Theorem 22. Let $T \in \overline{\mathcal{U}}\left(G^{\prime}\right)=\overline{\mathcal{U}}_{n}^{(p \nsim q)}$ and $\operatorname{root}(T)=r$. Consider the bijective map $\phi: \operatorname{PF}\left(G^{\prime}, r\right) \rightarrow \operatorname{SPT}\left(G^{\prime}, r\right)$ induced by Depth-First-Search algorithm and $\mathcal{P}_{T}$ is the unique $\left(G^{\prime}, r\right)$-parking function such that $\phi\left(\mathcal{P}_{T}\right)=T$. Let $\mathcal{M}_{G}=\left\langle m_{A}: \emptyset \neq A \subseteq[n]\right\rangle$ and $\mathcal{M}_{\left(G^{\prime}, r\right)}=\left\langle\bar{m}_{A}: \emptyset \neq A \subseteq[n] \backslash\{r\}\right\rangle$ be the parking function ideals. Suppose, if possible, $\mathcal{P}_{T} \neq \widehat{\mathcal{P}}$ for all $\mathcal{P} \in \operatorname{sPF}(G)$. Then $m_{[n]} \prod_{j \in[n] \backslash\{r\}} x_{j}^{\mathcal{P}_{T}^{(j)}}$ is not a standard monomial of $\mathcal{M}_{G}^{(n-2)}$. Thus there exists $\emptyset \neq A \subsetneq[n]$ such that $m_{A}$ divides $m_{[n]} \prod_{j \in[n] \backslash\{r\}} x_{j}^{\mathcal{P}_{T}^{(j)}}$.

Let $r \in A$ but $r \notin\{p, q\}$. As $m_{A} \mid m_{[n]} \prod_{j \in[n] \backslash\{r\}} x_{j}^{\mathcal{P}_{T}(j)}, x_{r}$ appearing in $m_{A}$ must have multiplicity 1 . Thus $A=[n]$, a contradiction. Now suppose $r=q \in A$ (or $r=$ $p \in A$ ). Then $A \neq[n]$ implies that $A=[n] \backslash\{p\}$ (respectively, $A=[n] \backslash\{q\}$ ). In fact, $m_{[n] \backslash\{p\}}=\left(\prod_{j \in[n] \backslash\{p, q\}} x_{j}^{2}\right) x_{q}$ and $m_{[n] \backslash\{q\}}=\left(\prod_{j \in[n] \backslash\{p, q\}} x_{j}^{2}\right) x_{p}$. Clearly, in either of the cases, $\bar{m}_{[n] \backslash\{p, q\}}=\prod_{j \in[n] \backslash\{p, q\}} x_{j}$ divides $\prod_{j \in[n] \backslash\{r\}} x_{j}^{\mathcal{P}_{T}(j)}$, a contradiction to $\mathcal{P}_{T}$ being $\left(G^{\prime}, r\right)$-parking function.

Finally, if $r \notin A$, then $\bar{m}_{A}=\frac{m_{A}}{\operatorname{gcd}\left(m_{A}, m_{[n]}\right)}$ and $\bar{m}_{A}$ divides $\prod_{j \in[n] \backslash\{r\}} x_{j}^{\mathcal{P}_{T}(j)}$. This shows that $\mathcal{P}_{T}$ is not a $\left(G^{\prime}, r\right)$-parking function, again a contradiction. This completes the proof.

We now determine conditions so that $\mathcal{U}_{n}^{(p \nsim q)}=\overline{\mathcal{U}}_{n}^{(p \nsim q)}$.

Proposition 24. $\mathcal{U}_{n}^{(p \nsim q)} \backslash \overline{\mathcal{U}}_{n}^{(p \nsim q)}=\left\{T \in \mathcal{U}_{n}^{(p \nsim q)}: \operatorname{root}(T)=p\right.$ and $\left.\mathcal{P}_{T}(q)=0\right\}$.

Proof. Let $T \in \mathcal{U}_{n}^{(p \nsim q)}$ such that $\operatorname{root}(T)=r \neq p$. Consider the unique $\left(G^{\prime}, r\right)$-parking function $\mathcal{P}_{T}$ such that $\phi\left(\mathcal{P}_{T}\right)=T$. As $T$ is uprooted, all the edges $(r, j)$ in $G^{\prime}$ for $j>r$ must get dampened. Thus $\mathcal{P}_{T}(j) \geqslant 1$ for all $j>r$ such that $r \sim j$ in $G^{\prime}$ or $G$. Since $G=K_{n+1}-\left\{e_{p, q}\right\}, T \in \overline{\mathcal{U}}_{n}^{(p \nsim q)}$.

Since there are no uprooted tree $T$ on the vertex set $[n]$ with $\operatorname{root}(T)=1$, it follows from Proposition 24 that $\mathcal{U}_{n}^{(p \nsim q)}=\overline{\mathcal{U}}_{n}^{(p \nsim q)}$ if and only if $p=1$. The following corollary is immediate.

Corollary 25. For $n \geqslant 3$ and $G=K_{n+1}-\left\{e_{1, n}\right\}$, the map $\phi_{G}: \operatorname{sPF}(G) \rightarrow \mathcal{U}_{n}^{(1 \nsim n)}=\mathcal{U}_{n}^{\prime}$ induces a bijection between the set of spherical $G$-parking functions and the set of uprooted trees on the vertex set $[n]$ with $1 \nsim n$. 
Remark 26. By renumbering vertices of $G$, we easily see that

$$
\left|\operatorname{sPF}\left(K_{n+1}-\left\{e_{p, q}\right\}\right)\right|=\left|\operatorname{sPF}\left(K_{n+1}-\left\{e_{1, n}\right\}\right)\right|=\left|\mathcal{U}_{n}^{\prime}\right|
$$

for any edge $e_{p, q}$ between vertices $p, q \in[n]$ with $p<q$. Thus, $\left|\overline{\mathcal{U}}_{n}^{(p \nsim q)}\right|=\left|\mathcal{U}_{n}^{\prime}\right|$.

The bijection $\phi_{n}: \operatorname{sPF}\left(K_{n+1}\right) \rightarrow \mathcal{U}_{n}$ constructed in Theorem 22 can be extended to the case of the complete multigraph $K_{n+1}^{a, b}$ on the vertex set $V$.

Let $\operatorname{sPF}\left(K_{n+1}^{a, b}\right)$ be the set of spherical $K_{n+1}^{a, b}$-parking functions. Let $\mathcal{U}_{n}^{b}$ be the set of uprooted tree $T$ on the vertex set $[n]$ with label $\ell: E(T) \rightarrow\{0,1, \ldots, b-1\}$ on the edges of $T$ and a weight $\omega(r) \in\{0,1, \ldots, b-1\}$ assigned to the root $r$ of $T$. Clearly, $\left|\mathcal{U}_{n}^{b}\right|=b^{n}\left|\mathcal{U}_{n}\right|=b^{n}(n-1)^{n-1}$. Also, $\left|\operatorname{sPF}\left(K_{n+1}^{a, b}\right)\right|=b^{n}(n-1)^{n-1}$ is independent of $a$. We may assume that $a \geqslant b$. As an application of the Depth-First-Search algorithm for multigraph (Theorem 9), we construct a bijection

$$
\phi_{n}^{b}: \operatorname{sPF}\left(K_{n+1}^{a, b}\right) \rightarrow \mathcal{U}_{n}^{b} .
$$

The reduced spherical $K_{n+1}^{a, b}$-parking function $\widetilde{\mathcal{P}}$ associated to $\mathcal{P} \in \operatorname{sPF}\left(K_{n+1}^{a, b}\right)$ is given by $\widetilde{\mathcal{P}}(i)=\mathcal{P}(i)-a$ for all $i \in[n]$. Let $\widetilde{\operatorname{sPF}}\left(K_{n+1}^{a, b}\right)=\left\{\widetilde{\mathcal{P}}: \mathcal{P} \in \operatorname{sPF}\left(K_{n+1}^{a, b}\right)\right\}$. Then as $a \geqslant b$, we can verify that $\widetilde{\operatorname{sPF}}\left(K_{n+1}^{a, b}\right) \subseteq \operatorname{PF}\left(K_{n+1}^{a, b}\right)$. Let $K_{n}^{b}=K_{n+1}^{a, b}-\{0\}$ be the complete multigraph on the vertex set $[n]$ such that $|E(i, j)|=b$ for every distinct pair $\{i, j\}$ of vertices.

Theorem 27. There exists a bijection $\phi_{n}^{b}: \operatorname{sPF}\left(K_{n+1}^{a, b}\right) \rightarrow \mathcal{U}_{n}^{b}$ such that

$$
\operatorname{rsum}(\mathcal{P})+\omega(r)+1=\kappa\left(K_{n}^{b}, T\right)+\sum_{e \in E(T)} \ell(e) \quad \text { for all } \quad \mathcal{P} \in \operatorname{sPF}\left(K_{n+1}^{a, b}\right),
$$

where $T=\phi_{n}^{b}(\mathcal{P})$ and weight $\omega(r) \in\{0,1, \ldots, b-1\}$ at the $\operatorname{root}(T)=r$.

Proof. Let $\mathcal{P} \in \operatorname{sPF}\left(K_{n+1}^{a, b}\right)$. Then $\widetilde{\mathcal{P}} \in \operatorname{PF}\left(K_{n+1}^{a, b}\right)$. Choose the largest vertex $r$ of $K_{n}^{b}=$ $K_{n+1}^{a, b}-\{0\}$ such that $\widetilde{\mathcal{P}}(r)<b$. We claim that $\widetilde{\mathcal{P}}(j)<b$ for some $j<r$. Otherwise, $\mathcal{P}(i) \geqslant a+b$, for all $i \in[n] \backslash\{r\}$, a contradiction to $\mathcal{P} \in \operatorname{sPF}\left(K_{n+1}^{a, b}\right)$. Now consider $r$ to be the root of the complete multigraph $K_{n}^{b}$ on the vertex set $[n]$. Then $\widehat{\mathcal{P}}=\left.\widetilde{\mathcal{P}}\right|_{[n] \backslash\{r\}}$ is a $\left(K_{n}^{b}, r\right)$-parking function. On applying the Depth-First-Search algorithm for multigraph (Theorem 9), we get $\phi(\widehat{P}) \in \mathcal{U}_{n}^{b}$ with root $r$ and weight $\omega(r)=\widetilde{\mathcal{P}}(r)$. The mapping $\phi_{n}^{b}: \operatorname{sPF}\left(K_{n+1}^{a, b}\right) \rightarrow \mathcal{U}_{n}^{b}$ given by $\phi_{n}^{b}(\mathcal{P})=\phi(\widehat{P})$ is clearly injective. Since $\left|\operatorname{sPF}\left(K_{n+1}^{a, b}\right)\right|=$ $\left|\mathcal{U}_{n}^{b}\right|=b^{n}(n-1)^{n-1}$, the map $\phi_{n}^{b}$ is a bijection. Also,

$$
g\left(K_{n}^{b}\right)-\sum_{i \in[n] \backslash\{r\}} \widetilde{\mathcal{P}}(i)=\operatorname{rsum}(\widehat{P})=\kappa\left(K_{n}^{b}, \phi(\widehat{P})\right)+\sum_{e \in E(\phi(\widehat{P}))} \ell(e) .
$$

Since $\operatorname{rsum}(\mathcal{P})=g\left(K_{n+1}^{a, b}\right)-\sum_{i \in[n]} \mathcal{P}(i)$, we verify that $\operatorname{rsum}(\widehat{P})=\operatorname{rsum}(\mathcal{P})+\omega(r)+1$. 


\subsection{Counting uprooted trees}

In this subsection, we determine the number $\left|\mathcal{U}_{n}^{\prime}\right|$ of uprooted trees on the vertex set $[n]$ with $1 \nsim n$. Let $\mathcal{T}_{n, 0}$ be the set of labelled trees on the vertex set $[n]$ such that the root has no child (or son) with smaller labels. Let $\mathcal{A}_{n}$ be the set of labelled rooted-trees on the vertex set $[n]$ with a non-rooted leaf $n$. Chauve, Dulucq and Guibert [1] constructed a bijection $\eta: \mathcal{T}_{n, 0} \rightarrow \mathcal{A}_{n}$. As earlier, let $\mathcal{U}_{n}$ be the set of uprooted trees on the vertex set $[n]$. Also, let $\mathcal{B}_{n}$ be the set of labelled rooted-trees on the vertex set $[n]$ with a non-rooted leaf 1 . We see that there are bijections $\mathcal{U}_{n} \rightarrow \mathcal{T}_{n, 0}$ and $\mathcal{B}_{n} \rightarrow \mathcal{A}_{n}$ obtained by simply changing label $i$ to $n-i+1$ for all $i$. The bijection $\eta: \mathcal{T}_{n, 0} \rightarrow \mathcal{A}_{n}$ induces a bijection $\psi: \mathcal{U}_{n} \rightarrow \mathcal{B}_{n}$. For sake of completeness, we briefly describe construction of the bijection $\psi$ essentially as in [1].

Let $T \in \mathcal{U}_{n}$ with root $r$. Note that $r \neq 1$.

Step (1) : Consider a maximal increasing subtree $T_{0}$ of $T$ containing 1 . Let $T_{1}, \ldots, T_{l}$ be the subtrees (with at least one edge) of $T$ obtained by deleting edges in $T_{0}$. Let $r_{i}$ be the root of $T_{i}$ for $1 \leqslant i \leqslant l$. The root $r$ of $T$ must be a root of one of the subtrees $T_{i}$. Let $r_{j}=r$. Then 1 is a leaf of $T_{j}$.

Step (2) : If $T_{0}$ has $m$ vertices, then $T_{0}$ is determined by an increasing tree $\overline{T_{0}}$ on the vertex set $[m]$ and a set $S_{0}$ of labels on $T_{0}$. We write $T_{0}=\left(\overline{T_{0}}, S_{0}\right)$.

Step (3) : Let $\overline{S_{0}}=\left(S_{0} \backslash\{1\}\right) \cup\{r\}$. Then $\left(\overline{T_{0}}, \overline{S_{0}}\right)$ determines an increasing subtree $\widetilde{T}_{0}$ with root $r^{\prime}=\min \left\{\overline{S_{0}}\right\}$. Graft $T_{j}$ on the increasing subtree $\widetilde{T}_{0}$ at the root $r$ and obtain a tree $T_{j}^{\prime}$. Now graft $T_{i}(i \neq j)$ on $T_{j}^{\prime}$ at $r_{i}$ and obtain a tree $T^{\prime}$ with root $r^{\prime}$. Also note that 1 is a non-rooted leaf of $T^{\prime}$.

All the above steps can be reversed, thus $\psi(T)=T^{\prime}$ defines a bijection $\psi: \mathcal{U}_{n} \rightarrow \mathcal{B}_{n}$.

Lemma 28. $\left|\mathcal{U}_{n}\right|=\left|\mathcal{B}_{n}\right|=(n-1)^{n-1}$.

Proof. The bijection $\psi: \mathcal{U}_{n} \rightarrow \mathcal{B}_{n}$ gives $\left|\mathcal{U}_{n}\right|=\left|\mathcal{B}_{n}\right|$. The number of labelled rootedtrees on the vertex set $\{2,3, \ldots, n\}$ by Cayley's formula is $(n-1)^{n-2}$. Any tree in $\mathcal{B}_{n}$ is obtained uniquely by attaching 1 to any node $i$ of a labelled rooted tree on the vertex set $\{2,3, \ldots, n\}$. Since there are exactly $n-1$ possibilities for $i$, we have $\left|\mathcal{B}_{n}\right|=(n-$ $1)^{n-2}(n-1)=(n-1)^{n-1}$.

For $n \geqslant 3$, let $\mathcal{U}_{n}^{\prime}=\left\{T \in \mathcal{U}_{n}: 1 \nsim n\right.$ in $\left.T\right\}$. We shall determine the image $\psi\left(\mathcal{U}_{n}^{\prime}\right) \subseteq \mathcal{B}_{n}$ of $\mathcal{U}_{n}^{\prime}$ under the bijection $\psi: \mathcal{U}_{n} \rightarrow \mathcal{B}_{n}$. Let $\mathcal{B}_{n}^{\prime}=\left\{T^{\prime} \in \mathcal{B}_{n}: 1 \nsim n\right.$ in $\left.T^{\prime}\right\}$. Set

$$
\begin{aligned}
\mathcal{A} & =\left\{T^{\prime} \in \mathcal{B}_{n}^{\prime}: \operatorname{root}\left(T^{\prime}\right)=r^{\prime}=n\right\}, \\
\mathcal{B}^{\prime} & =\left\{T^{\prime} \in \mathcal{B}_{n}^{\prime}: \operatorname{root}\left(T^{\prime}\right)=r^{\prime} \neq n \text { with } r^{\prime} \sim n \text { and } 1 \text { is a descendent of } n\right\}, \\
\mathcal{B}^{\prime \prime} & =\left\{T^{\prime} \in \mathcal{B}_{n}^{\prime}: \operatorname{root}\left(T^{\prime}\right)=r^{\prime} \neq n \text { with } r^{\prime} \nsim n\right\} .
\end{aligned}
$$

Lemma 29. $\psi\left(\mathcal{U}_{n}^{\prime}\right)=\mathcal{A} \coprod \mathcal{B}^{\prime} \coprod \mathcal{B}^{\prime \prime}$.

Proof. Let $T^{\prime} \in \mathcal{B}_{n}$. Then there is a unique $T \in \mathcal{U}_{n}$ such that $T^{\prime}=\psi(T)$. Let $r$ and $r^{\prime}$ be the roots of $T$ and $T^{\prime}$, respectively. Clearly, $r \neq 1$. Let $\operatorname{Son}_{T}(1)$ be the set of sons of 1 in $T$. Then from the construction of $T^{\prime}=\psi(T), r^{\prime}=\min \left\{\{r\} \cup \operatorname{Son}_{T}(1)\right\}$. Also, the leaf 1 
in $T^{\prime}$ is adjacent to $j$ if and only if $j=\operatorname{par}_{T}(1)$ is the parent of $1 \mathrm{in} T$. This shows that $1 \nsim n$ in $T$ if and only if $1 \nsim n$ in $T^{\prime}$. Hence, $\psi\left(\mathcal{U}_{n}^{\prime}\right) \subseteq \mathcal{B}_{n}^{\prime}$. Further, we see that $r^{\prime}=n$ if and only if 1 is already a leaf in $T$, and in this case, $T^{\prime}=\psi(T)=T$. In other words, $\mathcal{A} \subseteq \mathcal{U}_{n}^{\prime}$ and $\psi(T)=T$ for all $T \in \mathcal{A}$.

If $T^{\prime} \in \mathcal{B}^{\prime \prime}$, then the unique $T \in \mathcal{U}_{n}$ with $\psi(T)=T^{\prime}$ must have $1 \nsim n$ in $T$, that is, $T \in \mathcal{U}_{n}^{\prime}$. Now we consider the remaining case. Let $T^{\prime} \in \mathcal{B}_{n}^{\prime}$ with $\operatorname{root}\left(T^{\prime}\right)=r^{\prime} \neq n$ and $r^{\prime} \sim n$ in $T^{\prime}$. We shall show that $\psi(T)=T^{\prime}$ for $T \in \mathcal{U}_{n}^{\prime}$ if and only if 1 is a descendent of $n$ in $T^{\prime}$ (or equivalently, $T^{\prime} \in \mathcal{B}^{\prime}$ ). Consider the maximal increasing subtree $T_{0}^{\prime}$ of $T^{\prime}$ containing the root $r^{\prime}$. If 1 is a descendent of a leaf $r_{j}^{\prime}$ of $T_{0}^{\prime}$, then the maximal increasing subtree $T_{0}$ of $T$ containing 1 is obtained by replacing $r_{j}^{\prime}$ with 1 in the vertex set of $T_{0}^{\prime}$ and labeling it as indicated in Step (2) of the construction of $\psi$. Clearly, $r_{j}^{\prime}=r$ is the root of $T$. If $r_{j}^{\prime}=r \neq n$, then $1 \sim n$ in $T$ as $r^{\prime} \sim n$ in $T^{\prime}$. Thus, if $r_{j}^{\prime} \neq n$, i.e., 1 is not a descendent of $n$ in $T^{\prime}$, then $T^{\prime} \notin \psi\left(\mathcal{U}_{n}^{\prime}\right)$. On the other hand, if $r_{j}^{\prime}=n$, i.e., 1 is a descendent of $n$ in $T^{\prime}$ with $1 \nsim n$, then $\operatorname{root}(T)=r=n$ and $1 \nsim n$ in $T$.

Proposition 30. For $n \geqslant 3$, we have $\left|\mathcal{U}_{n}^{\prime}\right|=(n-1)^{n-3}(n-2)^{2}$.

Proof. By Lemma 29, we have $\left|\mathcal{U}_{n}^{\prime}\right|=\left|\psi\left(\mathcal{U}_{n}^{\prime}\right)\right|=|\mathcal{A}|+\left|\mathcal{B}^{\prime}\right|+\left|\mathcal{B}^{\prime \prime}\right|$. First we enumerate the subset $\mathcal{A}=\left\{T^{\prime} \in \mathcal{B}_{n}^{\prime}: \operatorname{root}\left(T^{\prime}\right)=r^{\prime}=n\right\}$. The number of labelled trees on the vertex set $\{2,3, \ldots, n\}$ with root $n$ is $(n-1)^{n-3}$. Since any tree in $\mathcal{A}$ is uniquely obtained by attaching 1 to any node $i \in\{2, \ldots, n-1\}$ of a labelled tree on the vertex set $\{2, \ldots, n\}$ with root $n$, we have $|\mathcal{A}|=(n-1)^{n-3}(n-2)$.

Let us consider the subset $\mathcal{C}=\left\{T^{\prime} \in \mathcal{B}_{n}^{\prime}: \operatorname{root}\left(T^{\prime}\right)=r^{\prime} \neq n\right\} \subseteq \mathcal{B}_{n}^{\prime}$. Clearly, $\mathcal{B}=\mathcal{B}^{\prime} \amalg \mathcal{B}^{\prime \prime} \subseteq \mathcal{C}$. The enumeration of $\mathcal{C}$ is similar to that of $\mathcal{A}$, except now the root $r^{\prime} \in\{2, \ldots, n-1\}$ can take any one of the $n-2$ values. Thus $|\mathcal{C}|=(n-1)^{n-3}(n-2)^{2}$. We can easily construct a bijective correspondence between $\mathcal{A}$ and $\mathcal{C} \backslash \mathcal{B}$. Let $T^{\prime} \in \mathcal{A}$. Then $1 \nsim n$ in $T^{\prime}$ and $\operatorname{root}\left(T^{\prime}\right)=n$. Consider the unique path from the root $n$ to the leaf 1 in $T^{\prime}$. As $1 \nsim n$ in $T^{\prime}$, the child $\tilde{r}$ of $n$ lying on this unique path is different from 1 . Let $\tilde{T}^{\prime}$ be rooted tree consisting of the tree $T^{\prime}$ with the new $\operatorname{root} \tilde{r}$. As $\operatorname{root}\left(\tilde{T}^{\prime}\right)=\tilde{r} \neq n, \tilde{r} \sim n$ and 1 is not a descendent of $n$ in $\tilde{T}^{\prime}$, we have $\tilde{T}^{\prime} \in \mathcal{C} \backslash \mathcal{B}$. The mapping $T^{\prime} \mapsto \tilde{T}^{\prime}$ from $\mathcal{A}$ to $\mathcal{C} \backslash \mathcal{B}$ is clearly a bijection. If $\tilde{T}^{\prime} \in \mathcal{C} \backslash \mathcal{B}$, then $\operatorname{root}\left(\tilde{T}^{\prime}\right)=\tilde{r} \neq n, \tilde{r} \sim n$ and 1 is not a descendent of $n$ in $\tilde{T}^{\prime}$. Now unique $T^{\prime} \in \mathcal{A}$ that maps to $\tilde{T}^{\prime}$ is the rooted tree obtained from $\tilde{T}^{\prime}$ by taking $n$ as the new root. Thus $|\mathcal{A}|=|\mathcal{C} \backslash \mathcal{B}|$ and hence, $\left|\mathcal{U}_{n}^{\prime}\right|=|\mathcal{C}|=(n-1)^{n-3}(n-2)^{2}$.

Theorem 31. Let $e_{p, q}$ be an edge of $K_{n+1}$ joining distinct vertices $p, q \in[n]$. For $n \geqslant 3$, the number of spherical parking functions of $K_{n+1}-\left\{e_{p, q}\right\}$ is given by

$$
\left|\operatorname{sPF}\left(K_{n+1}-\left\{e_{p, q}\right\}\right)\right|=\left|\mathcal{U}_{n}^{\prime}\right|=(n-1)^{n-3}(n-2)^{2} .
$$

Proof. In view of Theorem 23 and Remarks 26, the result follows.

Let $F_{l}=\left\{e_{1, n}, e_{1, n-1}, \ldots, e_{1, n-l+1}\right\}$ be a set of $l$-edges through the vertex 1 in the complete graph $K_{n+1}$. We consider the graph $K_{n+1}-F_{l}$ and ask the following question.

Question 32. What is the number of spherical $\left(K_{n+1}-F_{l}\right)$-parking functions?

Computations for smaller values of $n$ and $l$ indicate that

$$
\left|\operatorname{sPF}\left(K_{n+1}-F_{l}\right)\right|=(n-1)^{n-3}(n-l-1)^{2} .
$$




\section{Spherical $K_{m+1, n}$-parking functions}

Let $K_{m+1, n}$ be the complete bipartite graph on the vertex set $V^{\prime}=[0, m] \amalg[m+1, m+n]$, where $[0, m]=\{0,1, \ldots, m\}$ and $[m+1, m+n]=\{m+1, \ldots, m+n\}$. Let $K_{m+1, n}^{a, b}$ be the complete bipartite multigraph on $V^{\prime}$. More precisely, there are $a$ number of edges in $K_{m+1, n}^{a, b}$ between the root 0 and $j$, while $b$ number of edges between $i$ and $j$, where $i \in[m]$ and $j \in[m+1, m+n]$.

Proposition 33. We have $\left|\operatorname{sPF}\left(K_{m+1, n}^{a, b}\right)\right|=\left|\operatorname{sPF}\left(K_{n+1, m}^{a, b}\right)\right|$.

Proof. Let $E$ and $E^{\prime}$ be the set of all edges of $K_{m+1, n}^{a, b}$ and $K_{n+1, m}^{a, b}$ through the root 0 , respectively. On repeatedly applying the Lemma 17, we see that

$$
\left|\operatorname{sPF}\left(K_{m+1, n}^{a, b}\right)\right|=\left|\operatorname{sPF}\left(K_{m+1, n}^{a, b}-E\right)\right| \quad \text { and } \quad\left|\operatorname{sPF}\left(K_{n+1, m}^{a, b}\right)\right|=\left|\operatorname{sPF}\left(K_{n+1, m}^{a, b}-E^{\prime}\right)\right| .
$$

Since graphs $K_{m+1, n}^{a, b}-E$ and $K_{n+1, m}^{a, b}-E^{\prime}$ are obtained from each other by interchanging vertices as $i \leftrightarrow n+i$ and $m+j \leftrightarrow j$ (for $i \in[m], j \in[n]$ ), $\left|\operatorname{sPF}\left(K_{m+1, n}^{a, b}-E\right)\right|=$ $\left|\operatorname{sPF}\left(K_{n+1, m}^{a, b}-E^{\prime}\right)\right|$.

Although the root 0 is not connected to all the other vertices in the simple complete bipartite graph $K_{m+1, n}$, we can construct a map $\phi_{K_{m+1, n}}: \operatorname{sPF}\left(K_{m+1, n}\right) \rightarrow \mathcal{U}\left(K_{m, n}\right)$ as in Theorem 20, where $\mathcal{U}\left(K_{m, n}\right)$ is the set of uprooted spanning trees of $K_{m, n}=K_{m+1, n}-\{0\}$.

The reduced spherical $K_{m+1, n}$-parking function $\widetilde{\mathcal{P}}$ associated to $\mathcal{P} \in \operatorname{sPF}\left(K_{m+1, n}\right)$ is given by $\widetilde{\mathcal{P}}(j)=\mathcal{P}(j)$ for $1 \leqslant j \leqslant m$ and $\widetilde{\mathcal{P}}(j)=\mathcal{P}(j)-1$ for $m+1 \leqslant j \leqslant m+n$. We see that $K_{m, n}=K_{m+1, n}-\{0\}$ is the complete bipartite graph on the vertex set $[m] \coprod[m+1, m+n]$. The following statements can be easily verified.

(i) $\widetilde{\operatorname{sPF}}\left(K_{m+1, n}\right) \subseteq \operatorname{PF}\left(K_{m+1, n}\right)$.

(ii) Let $r=\max \{i \in[m+n]: \widetilde{\mathcal{P}}(i)=0\}$. Then $m+1 \leqslant r \leqslant m+n$.

(iii) $\widehat{\mathcal{P}}=\left.\widetilde{\mathcal{P}}\right|_{[m+n] \backslash\{r\}}$ is a $\left(K_{m, n}, r\right)$-parking function.

(iv) If $\phi: \operatorname{PF}\left(K_{m, n}, r\right) \rightarrow \operatorname{SPT}\left(K_{m, n}, r\right)$ is the bijection induced by Depth-First-Search algorithm, then $\phi(\widehat{\mathcal{P}})$ is an uprooted spanning tree of $K_{m, n}$.

Now define a map $\phi_{K_{m+1, n}}: \operatorname{sPF}\left(K_{m+1, n}\right) \rightarrow \mathcal{U}\left(K_{m, n}\right)$ given by $\phi_{K_{m+1, n}}(\mathcal{P})=\phi(\widehat{\mathcal{P}})$ for $\mathcal{P} \in \operatorname{sPF}\left(K_{m+1, n}\right)$. For each $T \in \mathcal{U}\left(K_{m, n}\right)$, let $\mathcal{P}_{T}$ be the unique $\left(K_{m, n}, r\right)$-parking function such that $\phi\left(\mathcal{P}_{T}\right)=T$. Let $\overline{\mathcal{U}}\left(K_{m, n}\right)=\left\{T \in \mathcal{U}\left(K_{m, n}\right): \mathcal{P}_{T}(j) \geqslant 1\right.$ for $\left.j>\operatorname{root}(T)\right\}$.

Theorem 34. The map $\phi_{K_{m+1, n}}: \operatorname{sPF}\left(K_{m+1, n}\right) \rightarrow \mathcal{U}\left(K_{m, n}\right)$ is injective with the image $\overline{\mathcal{U}}\left(K_{m, n}\right)$ and $\operatorname{sum}(\mathcal{P})=m(n-1)-\kappa\left(K_{m, n}, \phi_{K_{m+1, n}}(\mathcal{P})\right)+1$ for all $\mathcal{P} \in \operatorname{sPF}\left(K_{m+1, n}\right)$.

Proof. Proceed as in the proof of Theorems 20 and 22. 
Remark 35. The following three statements can be easily verified.

(1) $\left|\operatorname{sPF}\left(K_{m+1,1}\right)\right|=1=\left|\operatorname{sPF}\left(K_{1+1, n}\right)\right|$.

(2) Every spanning tree $T$ of $K_{m, n}$ with $\operatorname{root}(T)=m+n$ lies in $\overline{\mathcal{U}}\left(K_{m, n}\right)$. Thus

$$
\left|\left\{\mathcal{P} \in \operatorname{sPF}\left(K_{m+1, n}\right): \widetilde{\mathcal{P}}(m+n)=0\right\}\right|=\left|\operatorname{PF}\left(K_{m, n}\right)\right|=m^{n-1} n^{m-1} .
$$

(3) We have $\left|\operatorname{sPF}\left(K_{m+1, n}^{a, b}\right)\right|=b^{m+n}\left|\operatorname{sPF}\left(K_{m+1, n}\right)\right|$.

We could not enumerate $\operatorname{sPF}\left(K_{m+1, n}\right)$ or $\overline{\mathcal{U}}\left(K_{m, n}\right)$. Thus we ask the following question.

Question 36. What is the number of spherical $K_{m+1, n}$-parking functions?

For $n=2$, this question has an easy answer.

Proposition 37. For $m \geqslant 1,\left|\operatorname{sPF}\left(K_{m+1,2}\right)\right|=(m-1) 2^{m}+1$.

Proof. We know that $\left|\operatorname{sPF}\left(K_{m+1,2}\right)\right|=\left|\operatorname{sPF}\left(K_{m+1,2}-E\right)\right|$, where $E$ is the set of all edges of $K_{m+1,2}$ through the root 0 . Now the $m$-skeleton ideal of the (disconnected) graph $K_{m+1,2}-E$ is given by

$$
\mathcal{M}_{K_{m+1,2}-E}^{(m)}=\left\langle x_{i}^{2}, y_{j}^{m}, y_{1} y_{2}, x_{i_{1}} x_{i_{2}} \cdots x_{i_{s}} y_{j}^{m-s}: i \in[m] ; j=1,2 \text { and }\left\{i_{1}, \ldots, i_{s}\right\} \subseteq[m]\right\rangle,
$$

where $y_{j}=x_{m+j}$ for $j=1,2$. The standard monomials of $\mathcal{M}_{K_{m+1,2}-E}^{(m)}$ are of the forms $x_{i_{1}} x_{i_{2}} \ldots x_{i_{s}} y_{1}^{\alpha}$ with $0 \leqslant \alpha<m-s$ or $x_{i_{1}} x_{i_{2}} \cdots x_{i_{s}} y_{2}^{\beta}$ with $1 \leqslant \beta<m-s$. Thus the number of standard monomials of the first type is $\sum_{s=0}^{m}\left(\begin{array}{c}m \\ s\end{array}\right)(m-s)=m 2^{m-1}$, while that of the second type is $\sum_{s=0}^{m-1}\left(\begin{array}{c}m \\ s\end{array}\right)(m-s-1)=(m-2) 2^{m-1}+1$.

\section{Acknowledgements}

Thanks are due to the referee for helpful comments. The second author is thankful to the Ministry of Education, Government of India for financial support.

\section{References}

[1] C. Chauve, S. Dulucq and O. Guibert. Enumeration of some labelled trees. Proceeding of SFCA/FPSAC 2000 (Moscow, June 2000), D. Krobo and A. Mikhalev eds., Springer, pages 146-157, 2000.

[2] A. Dochtermann and R. Sanyal. Laplacian ideals, arrangements, and resolutions. J. Algebraic Combin., 40(3):805-822, 2014.

[3] A. Dochtermann. One-skeleta of $G$-parking function ideal : resolutions and standard monomials. arXiv:1708.04712v4, 2017.

[4] A. Dochtermann and W. King. Trees, parking functions, and standard monomials of skeleton ideals. arXiv:1806.04289v2, 2018.

[5] P. Gaydarov and S. Hopkins. Parking functions and tree inversions revisited. Adv. in Appl. Math., 80:151-179, 2016. 
[6] G. Kreweras. Une famille de polynômes ayant plusieurs propriétés énumeratives. Period. Math. Hungar., 11(4):309-320, 1980.

[7] A. Kumar and C. Kumar. Multigraded Betti numbers of multipermutohedron ideals. J. Ramanujan Math. Soc., 28(1):1-18, 2013.

[8] A. Kumar and C. Kumar. Alexander duals of multipermutohedron ideals. Proc. Indian Acad. Sci.(Math Sci.), 124(1):1-15, 2014.

[9] C. Kumar. Steck determinants and parking functions. Ganita, 68(1):33-38, 2018.

[10] M. Manjunath, F.-O. Schreyer and J. Wilmes. Minimal free resolutions of the $G$ parking function ideal and the toppling ideal. Trans. Amer. Math. Soc., 367(4):28532874, 2015.

[11] E. Miller and B. Sturmfels. Combinatorial commutative algebra. Graduate Texts in Mathematics 227, Springer-Verlag, New York, 2005.

[12] F. Mohammadi and F. Shokriech. Divisors on graphs, connected flags, and syzygies. Int. Math. Res. Not. IMRN, 24:6839-6905, 2014.

[13] D. Perkinson, Q. Yang and K. Yu. G-parking functions and tree inversions. Combinatorica, 37(2):269-282, 2017.

[14] J. Pitman and R. P. Stanley. A polytope related to empirical distributions, plane trees, parking functions, and the associahedron. Discrete and Computational Geometry, 27:603-634, 2002.

[15] A. Postnikov and B. Shapiro. Trees, parking functions, syzygies, and deformations of monomial ideals. Trans. Amer. Math. Soc., 356:3109-3142, 2004.

[16] R. P. Stanley. Enumerative combinatorics: volume 2. Cambridge Studies in Advanced Mathematics, 62. Cambridge University Press, 1999.

[17] C. H. Yan. On the enumeration of generalized parking functions. Proceedings of the 31-st Southeastern International Conference on Combinatorics, Graph Theory and Computing (Boca Raton, FL, 2000), Congressus Numerantium, 147:201-209, 2000.

[18] C. H. Yan. Parking functions. In Handbook of enumerative combinatorics, Discrete math. Appl. (Boca Raton), pages 853-893. CRC Press, Boca Raton, FL 2015. 